\title{
Establishing a Scale of Directional-Hemispherical Reflectance Factor I: The Van den Akker Method
}

\author{
William H. Venable, Jr., Jack J. Hsia, and Victor R. Weidner \\ Institute for Basic Standards, National Bureau of Standards, Washington, D.C. 20234
}

(May 22, 1977)

\begin{abstract}
A thorough study and error analysis was made of the Van den Akker or "auxiliary sphere" method of determining a scale of directional-hemispherical reflectance factor. The effects of a non-Lambertian distribution of the reflected radiation, including retroreflection, were included in this study. Three working standards were measured to an uncertainty in reflectance of less than \pm 0.0015 and these will be used as a basis for a new, more accurate NBS scale of $6^{\circ}$-hemispherical reflectance factor. The new scale and the NBS scale established in 1965 are in agreement to within the uncertainty of \pm 0.005 assigned to the 1965 scale.
\end{abstract}

Key words: Absolute reflectance; diffuse reflectance; error analysis; reflectance; reflectance factor; spectrophotometry.

\section{Foreword}

We have arranged this paper in a way which should accomodate readers who have an interest in this work from three quite different points of view:

1. Those who must make decisions based upon the results of the measurements are addressed principally in the Summary which directly follows this foreword. The material in parts I. and V.B. would also be of interest to such readers.

2. Those who wish to use the Van den Akker auxiliary sphere method for determining absolute reflectance should find the material in parts II, III, and V.A. to be of particular interest, and part IV can be read superficially, if at all, in order to glean a few technological ideas.

3. Those who are interested in a detailed understanding of the way in which these measurements are carried out at NBS and in a detailed discussion of the error analysis should, after a careful reading of parts II and III, place their emphasis on part IV.

\section{Summary}

Of the light or other optical radiation incident upon a surface in a given direction and at a given wavelength, a fraction is reflected from the surface. This fraction is called the spectral directional-hemispherical reflectance of the surface, and measuring it accurately is important in two different classes of applications:

1. Applications in which the value of the reflectance is of direct importance. Such applications include radiative energy transfer as in solar energy devices, lighting system engineering, calibrating radiometers in remote sensing satellites, and formulating the pigmentation in paints and other finishes.
2. Applications in which the actual value of the reflectance is of secondary importance, but for which the basis of measurement must be extremely stable in time. These applications include quality control in automated production and specifications involving color or appearance of finished products. For such applications, the instruments which are used are calibrated with material standards, and the laboratories supplying these standards must be able to measure reflectance directly in order to measure the standards and verify their stability.

The economic benefits derived from having this portion of the measurement system under control are very large, coming in the form of an accumulation of modest benefits over a very large base of application [1]. ${ }^{1}$ In order to realize these advantages, it is important that the uncertainty in the measurements be commensurate with the uniformity and stability of the reflectance of the surfaces encountered in practice.

For a number of applications, it is important to measure directional-hemispherical reflectance factors near 1 with an uncertainty in the neighborhood of \pm 0.001 . The present extensive work on spectral directional-hemispherical reflectance was undertaken at NBS for two reasons. First, the stated uncertainty of \pm 0.005 for the NBS reflectance factor scale established in $1965[2]^{1}$ was too large for many of the applications for which we were called upon to standardize the measurements. Second, and even more disturbing, intercomparisons between the scales of national standardizing laboratories in connection with the work of the International Standardization Organization (ISO) revealed differences as great as 0.015 between the measured value of reflectance of the same samples. Such a large difference can have serious economic consequences in international trade in finished goods such as paper.

As a first step in this work, we have investigated in great detail the Van den Akker auxiliary sphere method of determining spectral directional-hemispherical reflectance which

\footnotetext{
${ }^{1}$ Figures in brackets indicate the literature references on page 49.
} 
has been used in the past at NBS, improving upon the techniques and providing the necessary corrections to make the measurements precise and accurate to within \pm 0.0015 to the best of our knowledge. This step has been completed and is reported in this paper. The new and former NBS scales of measurement agree to well within the combined measurement uncertainties.

As a second step, we plan to investigate the techniques used by the other major national laboratories and to work with our colleagues in these laboratories to determine the cause of the discrepencies which have been encountered internationally. As part of this step, we have already completed a set of measurements using a second method of determination, the Sharp-Little method. The results obtained with that method are in good agreement with those reported in this paper and are to be published soon in another paper in this same series. We have visited the National Research Council Laboratories in Canada (NRC) for detailed discussions of these results. We also plan to conduct experiments with the Korte method currently used by the Physikalisch-Technische Bundesanstaldt (PTB) in Germany and possibly with one or two other approaches less commonly used.

The scale of directional-hemispherical reflectance as currently established will be disseminated through standards supplied through the NBS Office of Standard Reference Materials and through commercial secondary standards laboratories in the United States. The improvements in techniques which have been developed as a result of this work will be submitted to the appropriate committees of ASTM, ANSI, and TAPPI for possible incorporation in standard procedures. When the international discrepancies have been eliminated, we will be working with NRC, PTB, and possibly other laboratories as standardizing laboratories for ISO reflectance measurements.

\section{Introduction}

Directional-hemispherical $(d / h)$ reflectance factor measurements are important in a wide variety of applications. If the results of these measurements are to be a useful tool for technical communication, the measurements must be made accurately. Most reflectometers are not capable of measuring $d / h$ reflectance factor directly, but can only compare the reflectance factors of two objects. The calibration of such instruments is accomplished by measuring a standard object which has a known reflectance factor. To see that accurately measured reflectance standards are available to the measurement community is one of the primary responsibilities of the spectrophotometry group of the Radiometric Physics Section of the Institute for Basic Standards.

In the development and production quality control of finished products in which appearance is an important factor, the measurement of reflectance should be accurate to within \pm 0.002 . This level of accuracy or better is also important to rapidly evaluating the stability of reflecting materials under weathering and ageing. These two types of applications are the ones which commonly call for the lowest measurement uncertainty. It is difficult to produce highly reflecting surfaces for which the reflectance is reproduced to better than \pm 0.001 and the reflectance of most surfaces is not even uniform to this degree. Therefore, a reflectance measuring capability for which the uncertainty is less than \pm 0.001 is both necessary and sufficient for a national standardizing laboratory. The work described in this technical note is part of an effort to reduce the uncertainty in diffuse reflectance factor measurements at NBS from an estimated \pm 0.005 , which it has been in the recent past, to \pm 0.001 .

The National Bureau of Standards (NBS) has established its scale of $d / h$ reflectance factor in 1965 through an extensive series of measurements by Goebel, Caldwell, and Hammond [2]. At that time, Vitrolite [3] glass standards [4] to calibrate the General Electric Recording Spectrophotometer (GERS) [5] (Cat. 5962004 G28 No. 732986) were measured. Until recently, that instrument has been used for most reflectance measurements made at NBS. The reflectance of the Vitrolite standards has been shown to be very stable by measurements made over a period of thirty years relative to freshly prepared $\mathrm{MgO}$ surfaces [6]. In 1974, the scale of measurement was rechecked using the same apparatus used in the 1965 experiments and the agreement was within the experimental error associated with the measurements.

In April of 1974 and through the following year, it was determined from measurements made on a number of samples that there was a systematic difference between the scales of measurement being used by NBS and the National Research Council Laboratories of Canada (NRC). This difference was approximately 0.015 at the short wavelength end of the visible spectrum and decreased more or less regularly to approximately 0.01 at the long wavelength end of the spectrum (table I). A similar intercomparison between NRC and Physikalisch-Technische Bundesanstalt of Germany (PTB) [7] revealed only slight differences between the measurements being made by these laboratories (table II). Since the NBS uncertainty at that time is conservatively estimated to be \pm 0.005 and the NRC uncertainty is conservatively estimated to be \pm 0.003 , the difference is clearly significant.

TABLE I

Data from an intercomparison between NRC and NBS of reflectance measurements on a sprayed $\mathrm{BaSO}_{4}$ coating*. (June 1975) (Spectral directional $\left(6^{\circ}\right)$-hemispherical reflectance).

\begin{tabular}{cccc}
\hline \hline & & & Reflectance \\
Wavelength & NRC & NBS† & $\begin{array}{c}\text { Difference } \\
\text { (NRC-NBS) }\end{array}$ \\
\cline { 2 - 4 } & & & 0.014 \\
400 & 0.961 & 0.975 & .012 \\
440 & .963 & .975 & .012 \\
460 & .966 & .978 & .010 \\
480 & .970 & .980 & .011 \\
500 & .970 & .981 & .012 \\
520 & .972 & .981 & .013 \\
540 & .972 & .985 & .013 \\
560 & .972 & .985 & .010 \\
580 & .974 & .984 & .010 \\
600 & .975 & .985 & .010 \\
620 & .975 & .985 & .011 \\
640 & .976 & .982 & .009 \\
660 & .975 & .986 & .009 \\
680 & .976 & .985 & .008 \\
700 & .976 & .985 & .008 \\
720 & .977 & .985 & .007 \\
740 & .978 & .985 & .985 \\
\hline
\end{tabular}

* Samples prepared at NRC.

$\dagger$ Data from NBS test 232.14/49D.

The methods used by NBS, NRC, and PTB are all different. In any such case of disagreement between measurements, all measurements and methods are logically suspect 


\section{TABLE II}

Data from an intercomparison between NRC and PTB of reflectance measurements on a pressed $\mathrm{BaSO}_{4}$ tablet [7] (Spectral Hemisphericaldirectional $\left(0^{\circ}\right)$ reflectance factor).

\begin{tabular}{cccc}
\hline & \multicolumn{3}{c}{ Reflectance } \\
\cline { 2 - 4 } Wavelength & NRC & PTB & $\begin{array}{c}\text { Difference } \\
\text { (PTB-NRC) }\end{array}$ \\
\cline { 2 - 4 } 370 & 0.961 & 0.965 & 0.004 \\
380 & .969 & .971 & .002 \\
390 & .974 & .976 & .002 \\
400 & .977 & .979 & .002 \\
420 & .982 & .983 & .001 \\
440 & .985 & .984 & -0.001 \\
460 & .986 & .985 & -0.001 \\
480 & .987 & .986 & -0.001 \\
500 & .987 & .987 & 0 \\
550 & .988 & .988 & 0 \\
600 & .988 & .988 & 0 \\
650 & .987 & .988 & 0.001 \\
700 & .987 & .989 & .002 \\
750 & .987 & .988 & .001 \\
\hline
\end{tabular}

until the cause of the difference is located. However, since the NRC and PTB measurements were in close agreement, it seemed reasonable to investigate the method used by NBS first. A Diffuse Transmittance and Reflectance Reference Spectrophotometer [8], which will be referred to by the acronym DRS for convenience, was completed at NBS in October 1975. This has been used to make detailed measurements of all aspects of the Van den Akker auxiliary sphere method for realizing an absolute scale of $d / h$ reflectance factor [9], which was the method used to establish the NBS scale. The results of this investigation are reported in this paper.

As a result of this investigation a new NBS scale of diffuse reflectance factor measurements for $6^{\circ}$ incidence and hemispherical collection has been established which is believed to be accurate to within \pm 0.0015 . The new scale of reflectance factor differs by less than 0.002 from the scale formerly used by NBS. Since this investigation revealed no cause for the discrepancy between national laboratories, we are undertaking a thorough examination of the methods used by the other laboratories. The results of that work are to be reported in future papers.

\section{Definitions}

\section{A. Directional-Hemispherical Reflectance}

One quantity to be measured is the spectral $d / h$ reflectance, $\rho(\mathbf{U}, \mathbf{P}, \lambda)$ at a point on a plane surface. Using the notation described in NBS Technical Note 594-9 [10], this quantity can be expressed in terms of a generalized scattering function $S$ as:

$$
\rho(\mathbf{U}, \mathbf{P}, \lambda)=\iint S(\mathbf{U}, \mathbf{P} ; \mathbf{u}, \mathbf{p}, \lambda) \mathbf{u} \cdot d \mathbf{a} d \omega
$$

where $S(\mathbf{U}, \mathbf{P} ; \mathbf{u}, \mathbf{p}, \lambda)$ is the radiance emerging from the sample surface at point $\mathbf{p}$ in direction $\mathbf{u}$ due to a unit flux striking the sample at point $\mathbf{P}$ in direction $\mathbf{U}$. It is assumed that the sample is non-fluorescent so that all of the radiation can be confined to a very narrow band at wavelength $\lambda$. The integral with respect to the solid angle, $\omega$, in which the radiation emerges is taken over the entire hemisphere of directions and the integral with respect to the area a from which the radiance emerges is taken over the entire area from which the flux emerges. For the uniform, isotropic samples to be discussed in this paper, the $d / h$ reflectance can be represented by $\rho(\Gamma, \lambda)$, where $\Gamma$ is the angle between the direction of incidence $\mathbf{u}$ and the surface normal.

\section{B. Directional-Hemispherical Reflectance Factor}

A typical $d / h$ reflectometer has an entrance port which subtends at the sample a circularly shaped solid angle $\omega^{\prime}$ which in magnitude is on the order of $10^{-3}$ steradians. None of the flux falling into $\omega^{\prime}$ reaches the detector, and therefore the instrument does not compare $d / h$ reflectances of the samples as just defined but rather compares "directionalhemispherical" reflectance factors $[11] F(\Gamma, \lambda)$ for collection over the entire solid angle except for $\omega^{\prime}$. That is to say, the retroreflected flux is excluded from the measurement. To a very good approximation,

$$
F(\Gamma, \lambda)=\frac{\iint^{\prime} \mathrm{S}(\mathbf{U}, \mathbf{P} ; \mathbf{u}, \mathbf{p}, \lambda) \mathbf{u} \cdot d \mathbf{a} d \omega}{\iint^{\prime}[\delta(\mathbf{P}-\mathbf{p}) / \pi] \mathbf{u} \cdot d \mathbf{a} d \omega}
$$

where the prime on the integral indicates integration over the entire hemisphere except for $\omega^{\prime}$ and $\delta(\mathbf{P}-\mathbf{p}) / \pi$ is the idealized scattering function $S$ for an ideal diffuse reflector.

It is the quantity $F(\Gamma, \lambda)$ in which we are most interested when calibrating a reflectometer, and determining this quantity for several working standards is the object of the work reported in this paper.

\section{An Overview of the Method}

The method to be described for determining $d / h$ reflectance factor is more complex than the method for measuring $d / h$ reflectance originally proposed by Van den Akker. In the original method, it was assumed that $d / h$ reflectance is independent of the direction of incidence. Since this is not sufficiently correct for many surfaces, additional measurements are needed in order to accurately determine the $d / h$ reflectance using this approach. Also, the relationship between $d / h$ reflectance and the reflectance factor as measured by an instrument must be determined. However, for simplicity, we will continue to call the entire process the Van den Akker method.

Although this method is simple in principle and is potentially one of the most accurate methods, the descriptions of it in the literature are usually given in terms of involved summations of infinite series. These descriptions tend to cloud the simplicity of the method and to lead to a misunderstanding of it. Therefore, before describing the details of the measurements as performed at NBS, we will first provide a brief overview of the method in terms of four main steps. The first and last steps were used in the original Van den Akker method, and we have added the additional steps to take into account the variation of $\rho(\Gamma, \lambda)$ with $\Gamma$ and the difference between $\rho(\Gamma, \lambda)$ and $F(\Gamma, \lambda)$.

The primary measurement in the $V$ an den Akker method is a measurement of the reflectance of the wall of an integrating sphere (fig 1) under the irradiation it receives in the sphere. We will call this reflectance the $V$ an den Akker reflectance $\rho_{v}(\lambda)$. A flux is introduced into the sphere by reflecting a collimated beam of radiation from the back of the sphere. 


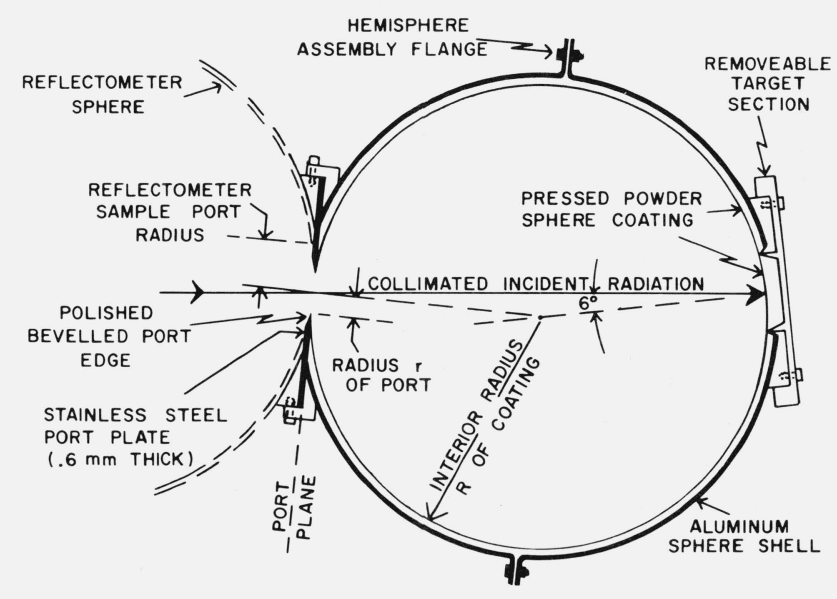

FiguRE 1. Sphere used for measuring Van den Akker reflectance.

Most of the radiation which strikes a given area of the sphere wall has undergone many reflections. In a sphere of this type, the average number of times a photon will have passed into the sphere wall and been reflected before it is absorbed or escapes would be between fifty and one-hundred. Because the reflection from the sphere wall is very diffuse, any asymmetry in the way the photons are introduced is dispelled in one or two reflections and the distribution of radiance, $L_{s}$, striking the wall has two characteristics properties. First, the irradiance is very nearly uniform over the sphere wall. This follows from the fact that a perfectly Lambertian (radiance constant with respect to direction) reflected flux would produce a uniform irradiance over the sphere wall. Since the distribution of the reflected flux is very nearly Lambertian, the distributon over the sphere wall of incident photons which have been reflected more than two or three times is for all practical purposes uniform. This phenomenon is well-known and forms the basis for the many applications of integrating spheres in which the sphere is used as a flux averaging device. Another property of the spherical geometry which is less often noticed is that a photon which leaves the sphere wall at an angle $\boldsymbol{\theta}$ will next strike the sphere wall at the same angle. Thus, after one or two reflections, an angular distribution of radiance $L_{s}(\Gamma)$, will be set up which represents, to within a proportionality constant, the incident and emerging radiance at any point on the sphere wall. Unlike the distribution of irradiance over the wall area, the distribution of the radiance with respect to the angle does not become uniform with angle but takes on a distribution determined by the bidirectional reflectance properties of the wall coating.

The remaining steps in the method relate the Van den Akker reflectance to the $d / h$ reflectance factor and provide a method of using this reflectance data to calibrate an instrument. The four steps in the Van den Akker method can be outlined as follows:

\section{A. Determining the Van den Akker Reflectance $\rho_{v}(\lambda)$}

A sphere with a single port (see fig. 1) is lined with a highly reflecting white coating, radiation is introduced into the port in a collimated beam, and, from measurements made of the flux re-emerging from the port and of the flux reflected from the target area, the $\mathrm{V}$ an den Akker reflectance $\rho_{v}{ }^{2}$ is determined. A flux $\phi_{0}(\lambda)$ is introduced into the sphere in a collimated beam which strikes the target area of reflectance $\rho_{t}(\Gamma, \lambda)$, providing a nearly Lambertian source of radiation which emits a flux $\phi_{0} \rho_{t}$. A fraction $\mathrm{f}$ of this flux passes out through the port as a nearly collimated beam, and the remaining portion falls on the sphere wall and is reflected with reflectance $\rho_{v}^{\prime}$, the average reflectance of the sphere wall, excluding the port. This flux, the strength of which is given by $\phi_{0} \rho_{t}(1-f) \rho^{\prime}{ }_{v}$, acts as a radiation source which is uniformly distributed over the sphere wall. This flux is the source of radiant energy for the total flux $\phi_{i}(\lambda)$ striking the sphere boundary. A fraction $f$ of $\lambda_{i}$ passes out through the port, and a fraction $\left(1-\rho_{v}^{\prime}\right)$ of the remaining $(1-f)$ is absorbed in the sphere wall or otherwise lost and is not returned to the sphere. In equilibrium, the flux lost from $\phi_{i}$ out the port and into the walls is replaced by the uniformly distributed source, from which an equilibrium flux balance equation can be obtained:

$$
\phi_{0} \rho_{t}(1-f) \rho_{v}^{\prime}=\phi_{i}\left[f+(1-f)\left(1-\rho_{v}^{\prime}\right)\right] .
$$

If the flux $\phi_{0}$ is the sample beam flux from a dual beam reflectometer, a signal $Q_{s}(\lambda)$ is obtained with the sphere in the sample position:

$$
Q_{s}=k\left[\phi_{i} f\left(1-f^{\prime}\right)+\phi_{0} \rho_{t} f\left(1-f^{\prime \prime}\right)\right]
$$

where $f^{\prime}$ is the fraction of the lambertian flux which escapes from the ports of the reflectometer sphere and $f^{\prime \prime}$ is the corresponding fraction of the nearly collimated beam which emerges from the sphere as a result of the first reflection from the back of the Van den Akker sphere. With the target area from the back of the sphere placed directly on the reflectometer sample port as a sample, a signal $Q_{t}(\lambda)$ is obtained:

$$
Q_{t}=k \phi_{0} \rho_{t}\left(1-f^{\prime}\right) .
$$

It is arranged that the angle of incidence $\Gamma$ at which $\phi_{0}$ strikes the target when $Q_{t}$ is measured is the same as when $Q_{s}$ is measured. Equations (3), (4), and (5) can be solved to obtain an expression for $\rho_{v}^{\prime}$ in terms of $Q_{t}, Q_{s}, f, f^{\prime}$ and $f^{\prime \prime}$.

$$
\rho_{v}^{\prime}=\frac{1-\left(f Q_{t} / Q_{s}\right)(1 /(1-\alpha))}{1-f}
$$

where

$$
\alpha=\frac{f\left(f^{\prime}-f^{\prime \prime}\right)}{\left(1-f^{\prime}\right)} \frac{Q_{t}}{Q_{s}} .
$$

If $\alpha$ were 0 , this would be the usual form cited for the Van den Akker reflectance. The factor $1 /(1-\alpha)$ takes into account that the first reflection of the incident beam from the target in the sphere emerges nearly collimated whereas the remaining flux emerging from the sphere is nearly Lambertian. If the reflectometer handled both fluxes in the same way, i.e., if $f^{\prime}$ and $f^{\prime \prime}$ were equal, this term would be zero. However, it is usually the cause that $f^{\prime \prime}$ is very much larger than $f^{\prime}$ and this contribution must be included.

\footnotetext{
${ }^{2}$ To keep the writing uncluttered, the functional dependence of each quantity is shown only when it is introduced and at key points in the development.
} 
An additional modification must be made in the original Van den Akker equation to take into account the effect on $\rho_{v}^{\prime}$ of the retroreflectance of the sphere wall. This modification, which results in only a small adjustment $\rho_{v}^{\prime}$ but which greatly complicates the form of eq (6), is discussed in detail in appendix A.

Since $(1-f)\left(1-\rho_{v}^{\prime}\right)$ in eq $(3)$ represents the portion of $\phi_{i}$ which is lost everywhere but out of the port, it follows that the reflectance $\rho_{v}{ }^{\prime}$ is the average reflectance over the sphere area excluding the port. To relate $\rho_{v}^{\prime}$ to a property of the sphere coating, it is important that the sphere coating be uniform and completely cover all of the sphere area except for the entrance port. If it is necessary to have cracks or other gross imperfections in the coating, the losses in such imperfections must be estimated and $\rho_{v}^{\prime}$ must be suitably corrected in order to obtain the Van den Akker reflectance $\rho_{v}$ of the sphere coating.

\section{B. Adjustment from Van den Akker Reflectance $\rho_{v}(\lambda)$ to d/h Reflectance $\rho(\Gamma, \lambda)$}

In order that the need for the next steps be more readily understood, $\rho_{v}$ will be interpreted in terms of the directionalhemispherical reflectance, $\rho$, as defined in eq (1).

$$
\rho_{v}=\frac{2 \pi \int_{0}^{\pi / 2} L_{s}(\Gamma) \rho(\Gamma) \cos (\Gamma) \sin (\Gamma) d \Gamma}{2 \pi \int_{0}^{\pi / 2} L_{s}(\Gamma) \cos (\Gamma) \sin (\Gamma) d \Gamma}
$$

where $L_{s}(\Gamma)$ is the radiance associated with $\phi_{i}$ at the incident angle $\Gamma$ and explicit indication of the dependence on wavelength has been omitted for clarity. As we have indicated, $\rho_{v}$ is just the weighted average over all directions of incidence $\Gamma$ of the $d / h$ reflectance. The weighting function $L_{s}(\Gamma) \cos \Gamma$ $\sin \Gamma$ is proportional to the irradiance of the sphere wall per unit angle at the angle $\Gamma$. If the $d / h$ reflectance were independent of angle of incidence, one can see from equation (7) that $\rho_{v}$ and $\rho(\Gamma)$ would be identical. However, in any real sphere coating, $\boldsymbol{\rho}(\boldsymbol{\Gamma})$ is not quite constant and this variation must be taken into account if $d / h$ reflectance is to be determined from $\rho_{v}$.

The flux, $\phi_{i}$, striking the sphere wall is composed of radiation most of which has undergone many reflections. As has already indicated, the radiance $L_{s}(\Gamma)$ striking the wall in one-location emerged from the wall at the same angle at some other location. When the irradiance of the wall is uniform and if the generalized scattering function $S$ of the coating were known, the function $L_{s}$ could be determined by solving the integral equation

$$
\rho_{v} L_{s}(\gamma)=2 \pi \int_{0}^{\pi / 2} L_{s}(\Gamma) S(\Gamma, \gamma) \cos \Gamma \sin \Gamma d \Gamma .
$$

Because the irradiance of the sphere wall is uniform, the self radiance distribution, $L_{s}$, can be determined to within a constant by measuring the radiance, $L_{s}(\gamma)$, emerging from the sphere coating through the port with an uncalibrated gonioreflectometer. The relative signal $N_{g}$ from the gonioreflectometer as a function of the angle of observation $\gamma$ can be related to $L_{s}(\gamma)$ as

$$
L_{s}(\gamma, \lambda)=k_{1}(\lambda) N_{g}(\gamma, \lambda) \cos (\gamma)
$$

where $k_{1}$ is a constant of proportionality.

To complete the adjustment, information about the form of $\rho(\Gamma, \lambda)$ is obtained. A flat sample of the sphere wall coating is prepared in the same way as the sphere wall coating and its relative $d / h$ reflectance is measured. The corrected reflectometer signal [12] $N_{G}$ is proportional to the $d / h$ reflectance.

$$
\rho(\Gamma, \lambda)=k_{2}(\lambda) N_{G}(\Gamma, \lambda) .
$$

The constant $k_{2}$ can be expressed in terms of measured quantities by simultaneous solution of eqs (7), (9), and (10).

$$
k_{2}(\lambda)=\frac{\rho_{v}(\lambda) \int N_{g}(\Gamma, \lambda) \sin \Gamma d \Gamma}{\int N_{G}(\Gamma, \lambda) N_{g}(\Gamma, \lambda) \sin \Gamma d \Gamma} \equiv \frac{\rho_{v}(\lambda)}{\bar{N}_{G}(\lambda)}
$$

where $\bar{N}_{G}$ is the weighted average of $N_{G}(\Gamma, \lambda)$ with weighting function $N_{g}(\Gamma, \lambda) \sin \Gamma$. With the value of $k_{2}$ determined, the $d / h$ reflectance for any wavelength and angle of incidence can be calculated from the relative $d / h$ reflectance data by using eq (10). However, we prefer to use an expression for $\rho(\Gamma, \lambda)$ which takes the form of a small adjustment of the Van den Akker reflectance. By substituting the expression for $k_{2}$ from eq (11) into eq (10) and using some algebraic manipulation, one can put eq (10) into the form

$$
\rho(\Gamma, \lambda)=\rho_{v}(\lambda)[1+C(\Gamma, \lambda)]
$$

where

$$
C(\Gamma, \lambda)=\frac{N_{G}(\Gamma, \lambda)}{\bar{N}_{G}(\lambda)}-1
$$

\section{From $d / h$ Reflectance $\rho(\Gamma, \lambda)$ to $d / h$ Reflectance Factor $F(\Gamma, \lambda)$}

If the generalized scattering function $S$ for directions of incidence and reflectance both falling within the reflectometer entrance port (retroreflectance at entrance angle $\Gamma$ ) were equal to the average of $S$ over all directions of viewing, then the reflectance $\rho(\Gamma, \lambda)$ and the reflectance factor $F(\Gamma, \lambda)$ will be equal. However, for the usual type of sphere coating, $S$ in the retroreflective direction has been observed to be as much as 50 percent above the average at small angles of observation [13]. Therefore, the bidirectional reflectance factor $F(\mathbf{U}$, $\mathbf{u} \lambda$ ) must be determined over the extent of the solid angle $\omega^{\prime}$ surrounding the incident direction $\mathbf{U}$ and the relationship between $F(\Gamma, \lambda)$ and $\rho(\Gamma, \lambda)$ determined from eqs (1) and (2) as

$$
F(\Gamma, \lambda)=\frac{\rho(\Gamma, \lambda)-\frac{1}{\pi} \int \mathbf{F}(\mathbf{U}, \mathbf{u}) \cos \gamma d \omega}{\left(1-\frac{1}{\pi} \omega^{\prime} \cos \gamma\right)}
$$

where the integral in $\omega$ is taken over $\omega^{\prime}$. (Note that $F(\mathbf{U}, \mathbf{u})=$ $\left.\pi \int S(\mathbf{U}, \mathbf{u}) d a.\right)$ 


\section{Determining the Directional-Hemispherical Reflec- tance Factor of Standards and Other Samples}

The $d / h$ reflectance factor $F(\Gamma, \lambda)$ as determined in the preceding four steps is the $d / h$ reflectance factor of the sphere wall coating. The final step is to use this information to measure a stable standard reflector which can then be used to calibrate a $d / h$ reflectometer. One of the methods used by Van den Akker [9] was to compare the standard directly with sections of the sphere wall which were hoped to be representative. Another method is to prepare a large number of samples using the same materials and preparation techniques that were used in coating the sphere itself and comparing these to the standard. In either case, the $d / h$ reflectance factor $F_{c}(\Gamma, \lambda)$ of the standard is calculated from the previously determined $d / h$ reflectance factor $F(\Gamma, \lambda)$ of the sphere wall as

$$
F_{c}(\Gamma, \lambda)=F(\Gamma, \lambda) \frac{n Q_{c}(\Gamma, \lambda)}{\sum_{i=1}^{n} Q_{i}(\Gamma, \lambda)}
$$

where $Q_{c}$ is the reflectometer signal for the standard and $Q_{i}$ is the reflectometer signal for the $i$ th one of the $n$ samples which are taken to represent the sphere wall. In day-to-day use, the $d / h$ reflectance factor $F_{x}(\Gamma, \lambda)$ of a sample can be measured by comparison to the stable standard.

$$
F_{x}(\Gamma, \lambda)=F_{c}(\Gamma, \lambda) \frac{Q_{x}(\Gamma, \lambda)}{Q_{c}(\Gamma, \lambda)}
$$

where the reflectometer readings $Q_{x}$ and $Q_{c}$ are taken close together in time in order to minimize the effects of instrument drift.

\section{The Measurements as Currently Made at NBS}

The overview in the preceding section describes in general the way that $d / h$ reflectance can be determined by the Van den Akker method. Three determinations of the $d / h$ reflectance of a set of standards has been made using the DRS. Through the experience gained in these determinations, a procedure for making the measurements with this instrument has been worked out. That procedure is described in this section to give a documented starting point for future improvements in the method and the data from these three determinations provide the basis for the current NBS scale of $6^{\circ} / h$ reflectance factor.

Three different materials were used as sphere coatings for the determinations. In the first determination, the sphere was coated with $\mathrm{BaSO}_{4}$ powder $[3,14]$ from a stock which had been on our laboratory shelf for some time and had been opened on previous occasions. This was used to obtain experience with the method and to obtain an idea of some of the difficulties which might be encountered with a material which was slightly contaminated and which had a slightly lower reflectance than the best quality coatings. In the second determination, the sphere was coated with Halon [3, 15] powder, and in the third determination a fresh lot of reagent grade $\mathrm{BaSO}_{4}$ powder $[3,16]$ was used. The advantages and disadvantages of each coating will be brought out in the discussion of the measurement errors in the description of each of the three main measurement steps.

\section{A. Determining the Van den Akker Reflectance $\rho_{v}(\lambda)$}

The design of the spheres used to determine the Van den Akker reflectance of the sphere coating material is shown in figure 1 . In some of his earlier work using this method, Van den Akker used a sphere with many removable sections in order to sample at many points the actual sphere coating being measured. However, the presence of many removable sections provides an uneven substructure for the sphere coating, creating a potential for dark rings and even cracks in the coating at the border of each removable section. Therefore, we decided to have only the target area removable and to rely on reproducing the coating for a representative sampling.

The sphere coating is made by packing the powder into the aluminum sphere shell with an electrically driven hammer which has a Teflon [3] head (fig. 2). The hammer is mounted on a stand and each hemisphere of the sphere is mounted in turn on a gimbal which allows it to be rotated about a point a fixed distance behind the hammer. In this way, a hemisphere of constant interior radius $R$ is packed uniformly in a mechanical way. A plastic ring attached to the flange of each hemisphere as it is being packed allows the packing jig to be aligned the same for both hemispheres and allows the coating to be built up to full thickness at the edge of the hemisphere. In this way, when the plastic ring is removed and the sphere is joined, a uniform sphere coating of interior radius $R$ results which has no crack at the plane where the hemispheres join.

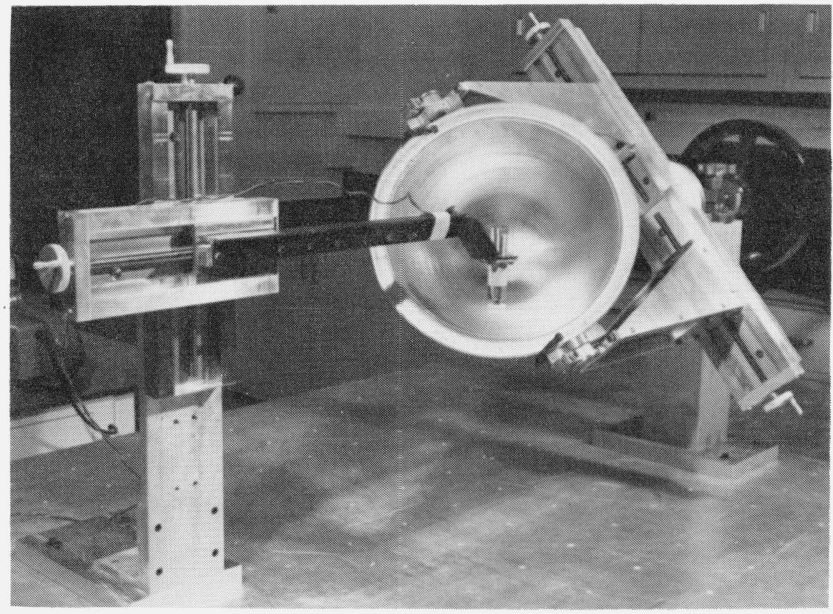

FIGURE 2. Apparatus used to produce the sphere coating.

\section{Determining the Uncorrected Van den Akker Reflectance $\boldsymbol{\rho}_{v}^{\prime}$}

The fraction $f$ introduced in eq (6) is taken as that portion of the area of the sphere of radius $R$ which is occupied by the port of radius $r$, i.e.,

$$
f=\left(1-\left(1-(r / R)^{2}\right)^{1 / 2}\right) / 2 .
$$

Associated with each determinaton of $f$ there is an estimated random error, as defined in appendix B. A random error $\delta R$ arises from our inability to pack the sphere to the same radius 
$R$ from one determination of reflectance to the next and from the inability to measure the average $R$ exactly. This gives rise to a random error $\delta f$ which is given by:

$$
\delta f=\frac{\left(r^{2} / R^{3}\right)}{2\left(1-(r / R)^{2}\right)^{1 / 2}} \delta R .
$$

A systematic error arises from any error in measuring $r$ and from assuming that the measured $r$ is the effective $r$. Both of these can be taken into account in terms of an uncertainty $\Delta r$ in the effective port radius. The estimated systematic error $\Delta f$ is given by

$$
\Delta f=\frac{\left(r / R^{2}\right)}{2\left(1-(r / R)^{2}\right)^{1 / 2}} \Delta r .
$$

The reflectance of the sphere was measured using the DRS with the $6^{\circ} / h$ (specular included) general purpose integrating sphere [17]. In this mode of operation, the instrument is a dual beam reflectometer with a capability of highly accurate measurements of relative $6^{\circ} / \mathrm{h}$ reflectance factor, i.e., the instrument measures a quantity $Q$ which is proportional to the $6^{\circ} / \mathrm{h}$ reflectance factor of the sample appearing in the plane of its sample port. With the sphere in place as a sample, a reflectance value which will be called $Q_{s}^{\prime}$ is measured. As is described in detail in the Technical Note on the DRS [18], a correction must be made for the small amount of radiation which does not enter the sphere port but instead is reflected from that portion of the sphere port plate which shows in the reflectometer sample port or is reflected from the sphere wall of the reflectometer itself. In order to correct for this scattered radiation, a second reflectance measurement is made with the auxiliary sphere port plate in place without the sphere behind it, i.e., with most of the sample beam of the reflectometer passing out into the room and being lost. The reflectance value $Q_{p}$ obtained in this manner must be subtracted from $Q_{s}^{\prime}$ in order to obtain a reflectance value proportional to the radiation being returned from inside the sphere. Most of the radiation emerging from the sphere passes freely back through the port into the reflectometer sphere. A small fraction of it, however, will strike the polished beveled edge of the auxiliary sphere port and will, thereby, suffer a slight loss as it returns to the reflectometer sphere. To correct for this loss, we measured the relative reflectance $Q_{f}$ of a flat plate of the same stainless steel from which the auxiliary sphere port plate was made. Since $Q_{f}$ in the reflectometer is approximately the reflectance of the sample relative to the sphere wall which in turn has a reflectance nearly $1, Q_{f}$ was approximately the reflectance of the stainless steel plate for $6^{\circ}$ incidence. The radiation coming from the sphere which strikes the beveled edge strikes it at near grazing incidence. Since the reflectance from a metal surface near grazing incidence is higher than the near-normal reflectance, we chose to represent the reflectance from the beveled edge by (1 $\left.+2 Q_{f}\right) / 3$. This estimate is based on the general shape of the reflectance curves for metals [19] and on the port dimensions (fig. 3) [20]. Under this assumption, if the projected solid angle subtended by the beveled edge of the port averaged over the port area is $\omega_{e}$, an expression for the corrected sphere reflectance $Q_{s}$ can be written as

$$
Q_{s}=\left(Q_{s}^{\prime}-Q_{p}\right) /\left[1-\frac{\omega_{e}}{\pi} \frac{2\left(1-Q_{\boldsymbol{f}}\right)}{3}\right] .
$$

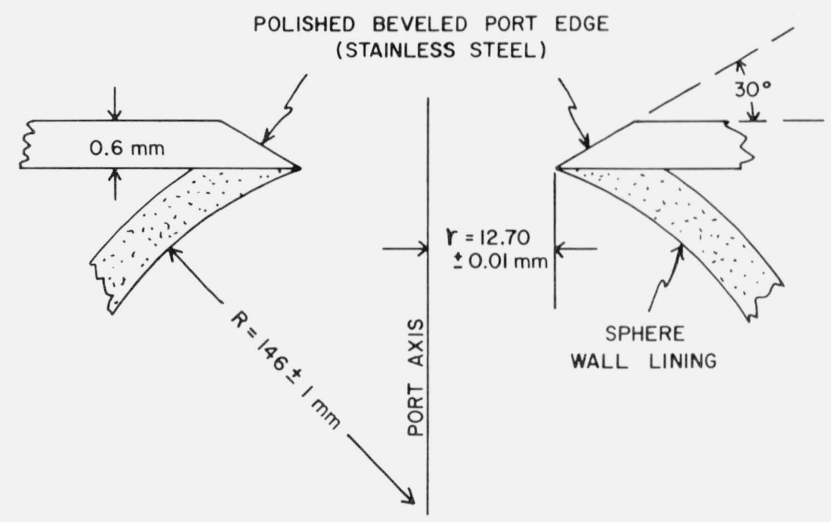

Figure 3. Cross section detail of polished beveled port edge.

Associated with this determination of $Q_{s}$, there is a random error $\delta Q_{s}$ arising from the random errors in measuring $Q_{s}^{\prime}$, $Q_{p}$, and $Q_{f}$. Because of the smallness of $\omega_{e}$, the denominator in eq (19) is approximately 1 and the small random error in $Q_{f}$ has little effect on the total random error. Therefore

$$
\delta Q_{s} \simeq\left(\delta Q_{s}^{\prime 2}+\delta Q_{p}^{2}\right)^{1 / 2} .
$$

Also associated with the determination of $Q_{s}$ is a systematic error $\Delta Q_{s}$ which arises from the uncertainty in $\omega_{e}$ and from the approximation used in estimating the effective reflectance of the beveled edge of the port. The systematic uncertainty in the beveled edge correction is estimated to be one-half as large as the correction itself, so that

$$
\Delta Q_{s} \simeq Q_{s} \frac{2\left(1-Q_{f}\right)}{3 \pi}\left(\Delta \omega_{e}^{2}+\left(\frac{\omega_{e}}{2}\right)^{2}\right)^{1 / 2}
$$

The reflectance of the removable target was measured using the same instrument. In this case a value $Q_{t}^{\prime}$ was obtained which also included a small contribution due to stray radiation from the sample beam which is reflected from the reflectometer sphere wall and from that portion of the target which is outside the region occupied by the auxiliary sphere port. A correction for this contribution was obtained by measuring $Q_{e}$ with a sample in the port made of the same material as the target but which has a hole in it the size of the port in the auxiliary sphere. Note that in this measurement, the beveled edge is not present, since the stainless steel port structure depicted in figure 3 is part of the auxiliary sphere. The corrected relative reflectance of the target $Q_{t}$ is obtained as

$$
Q_{t}=Q_{t}^{\prime}-Q_{e}
$$

An expression for the random error associated with this determination of $Q_{t}$ is determined in a straightforward fashion and resembles eq (20).

$$
\delta Q_{t}=\left[\delta Q_{t}^{\prime 2}+\delta Q_{e}^{2}\right]^{1 / 2} .
$$

The values of $f, Q_{s}$, and $Q_{t}$ obtained above were used in eq (6) (more specifically, eq (A7)) to calculate values of $\rho_{v}^{\prime}(\lambda)$, 
the Van den Akker reflectance of the sphere wall in its entirety. The random error in $\rho_{v}^{\prime}$ is obtained from eq $(6)$ as

$$
\begin{aligned}
\delta \rho_{v}^{\prime}=\left[\left(\left(1-Q_{t} / Q_{s}\right) /(1-f)^{2}\right)^{2} \delta f^{2}\right. & \\
& +\left(\left(f / Q_{s}\right) /(1-f)\right)^{2} \delta Q_{t}^{2} \\
& \left.\quad+\left(\left(f Q_{t} / Q_{s}^{2}\right) /(1-f)\right)^{2} \delta Q_{s}^{2}\right]^{1 / 2} .
\end{aligned}
$$

The systematic error in $\rho_{v}^{\prime}$ is given similarly by:

$$
\begin{aligned}
\Delta \rho_{v}^{\prime}=\left[\left(\left(1-Q_{t} / Q_{s}\right) /(1\right.\right. & \left.-f)^{2}\right)^{2} \Delta f^{2} \\
& \left.+\left(\left(f Q_{t} / Q_{s}^{2}\right) /(1-f)\right)^{2} \Delta Q_{s}^{2}\right]^{1 / 2}
\end{aligned}
$$

where $\Delta Q_{s}$ is only that portion of the systematic uncertainty in $Q_{s}$ given by eq $(21)$. Note that the contribution to the systematic error in $Q_{s}$ and $Q_{t}$ due to systematic errors in the DRS are omitted because these errors are very small [21] and because they tend to cancel when the ratio $Q_{t} / Q_{s}$ is taken in determining $\rho_{v}^{\prime}$ from eq (6). Therefore there is no $\Delta Q_{t}$ term in eq (25).

\section{From Uncorrected Van den Akker Reflectance $\rho_{v}^{\prime}$ to Corrected Van den Akker Reflectance $\boldsymbol{\rho}_{v}$}

The quantity $\rho_{v}^{\prime}$ as determined in the preceding section is the average $V$ an den Akker reflectance of the sphere wall. In arder to obtain the Van den Akker reflectance of a thick coating of the sphere wall material, it is necessary to correct $\rho_{v}^{\prime}$ for the effect of the crack around the removable target section and for translucency in the sphere wall coating.

a. The Gap Around the Target

Since the target portion of the sphere is removable, there is a narrow gap in the sphere wall around the target. The gap acts as a light pipe between two aluminum surfaces, so that the effective reflectance of the gap is essentially zero. Therefore, in order to relate the measured Van den Akker reflectance $\rho_{v}^{\prime}$ to the Van den Akker reflectance $\rho_{v}$ of the wall coating, the loss in the gap must be accounted for:

$$
\rho_{v}=\frac{\rho_{v}^{\prime}}{k_{t}\left[1-2 r_{t} W /\left(4 R^{2}-r^{2}\right)\right]}
$$

where $r_{t}$ is the radius of the target, $W$ is the width of the gap, $r$ is the radius of the entrance port, and $k_{t}$ is a transluency correction factor as determined in section IV. A.2.b. below. The systematic error associated with the crack correction is estimated to be one-half the magnitude of the correction. The total systematic error in $\rho_{v}$ is given by

$$
\Delta \rho_{v}=\rho_{v}\left[\left(\frac{\Delta \rho_{v}^{\prime}}{\rho_{v}^{\prime}}\right)^{2}+\left(\frac{\Delta k_{t}}{k_{t}}\right)^{2}+\left(\frac{r_{t} W}{4 R^{2}-r^{2}}\right)^{2}\right]^{1 / 2} .
$$

\section{b. Translucency of the Wall Coating}

The relative reflectance as a function of thickness was measured at each of three wavelengths on samples of each type of coating material. The results of these measurements are depicted in figure 4 . The coating in the sphere is, on the average, about $4 \mathrm{~mm}$ thick, so that the reflectance of a barium sulfate sphere wall is equal to the reflectance in the limit of a very thick wall, $\rho_{\infty}[22]$. Therefore, for these coatings $k_{t}=1$. However, in the case of Halon, the wall is not thick enough and a correction was made based on the data in figure 4 . In this correction, the thickness of the coating at any point was taken to be the distance from the surface to the aluminum substrate at that point, and a translucence correction factor $k_{t}$ was obtained by a calculation having the following form:

$$
k_{t}=\frac{\int \frac{\rho_{x}}{\rho_{10}} d A}{\int d A}
$$

where $\rho_{x}$ is the relative reflectance for the coating thickness at a given point,

$\rho_{10}$ is the relative reflectance for a $10 \mathrm{~mm}$ thick coating (assumed equal to $\rho_{\infty}$ ),

$d A$ is an element of the sphere wall area, and the integral is taken over the entire area of the sphere wall.

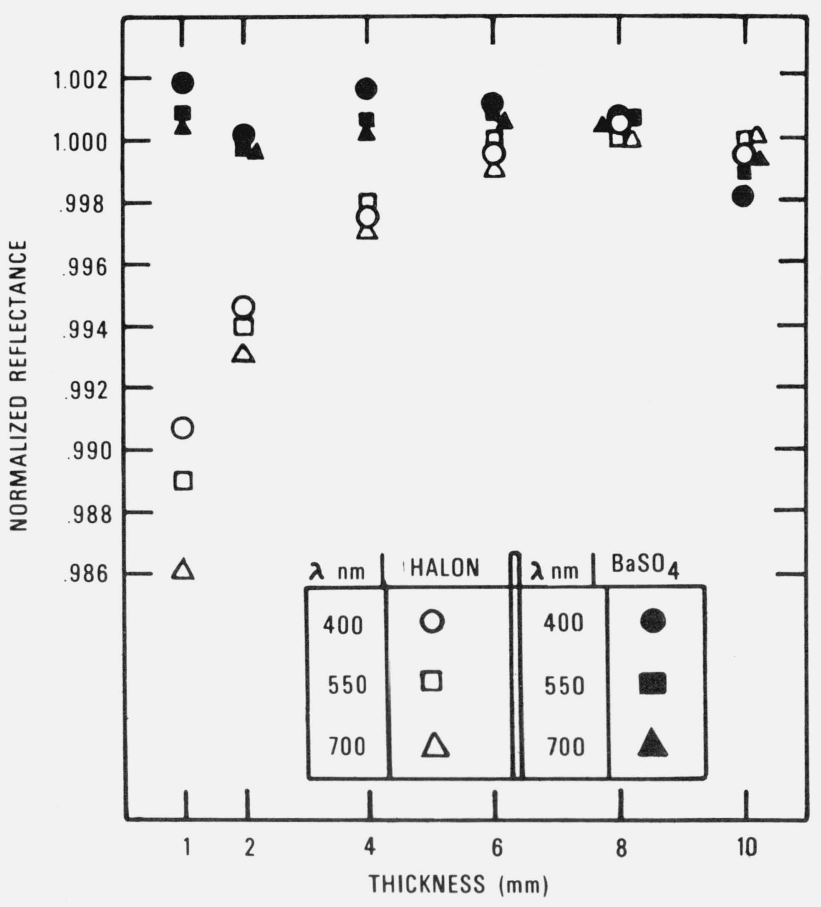

FigurE 4. Reflectance of wall coatings as function of thickness. (Normalized to give a reflectance of approximately 1 for thick samples.)

It is estimated that the coating thickness measurements might have been in error by as much as $1 / 2 \mathrm{~mm}$ for the $4 \mathrm{~mm}$ thickness. Therefore the calculation of eq (28) was redone using 3.5 and $4.5 \mathrm{~mm}$ for the average coating thickness over the main sphere wall in order to obtain an estimate of the random uncertainty $\delta k_{t}$ of this correction. An expression for 
the random error in $\rho_{v}$ as given by eq (26) is

$$
\delta \rho_{v}=\rho_{v}\left[\left(\frac{\delta \rho_{v}^{\prime}}{\rho_{v}^{\prime}}\right)^{2}+\left(\frac{\delta k_{t}}{k_{t}}\right)^{2}\right]^{1 / 2}
$$

Since using the distance from the coating surface to the aluminum substrate as its thickness is only a rough approximation in the regions of the port and of the target boundary, a systematic error will be introduced by that approximation. An estimation of this uncertainty was obtained by calculating $k_{t}^{\prime}$ $=\rho_{4} / \rho_{10}$, and estimating the systematic error as $\left(k_{t}^{\prime}-k_{t}\right) / 2$. We assumed the translucency correction to be approximately independent of wavelength. The data in figure 4 , for $1 \mathrm{~mm}$ thickness indicates that this may not be exactly correct. Therefore, there may be a wavelength dependent systematic error of approximately \pm 0.0005 in $k_{t}$, with the positive error associated with short wavelengths and the negative error with long wavelengths.

The Halon coatings used in our apparatus had a density of $0.8 \mathrm{~g} \mathrm{~cm}^{-3}$. Grum [23] refers to a coating of Halon which is opaque at $2 \mathrm{~mm}$ thickness. However, from the description of the pressure under which the coating was formed, his coatings are probably much denser than ours.

\section{Sample Curvature and Sphere Size}

Questions have been raised concerning the effect of the size of the sphere upon the Van den Akker reflectance. Possible errors may arise due to the inability of the measuring instrument to properly compare the reflectance of curved and flat surfaces, either due to the difference in the relative areas of the port and the remainder of the sphere wall or due to a change in the reflectance of the wall coating with curvature. This question has been addressed experimentally in two different ways. The relative $6^{\circ} / h$ reflectance of curved and flat samples was measured directly. The average relative reflectance at $550 \mathrm{~nm}$ of four concave $\mathrm{BaSO}_{4}[14]$ samples from the first determination was $0.9826 \pm 0.0027$ and the average reflectance of two flat samples of the same material was found to be 0.9834 , and the uncertainty is probably of the same order of magnitude. The difference of 0.0008 is probably not significant.

In the second test, the Van den Akker reflectance was determined using two different sized spheres, one with a 95 $\mathrm{mm}$ interior radius and the other with a $146 \mathrm{~mm}$ interior radius. Both spheres were coated with the type of $\mathrm{BaSO}_{4}[6]$ used in the third determination. The Van den Akker reflectances for these two determinations are given in table III. It can be seen that there is no significant difference between the results from the two spheres.

\section{Results and Error Analysis}

The Van den Akker reflectances $\rho_{v}$ determined for each coating at twenty-five nanometer intervals over the wavelength range $400 \mathrm{~nm}$ to $750 \mathrm{~nm}$ are given in tables IV,V and VI, and are pictured in figure 5. A listing of typical values used in the calculations for the Van den Akker reflectance is shown in table VII, and representative calculations of the random and systematic uncertainties in $\rho_{v}$ are presented in tables VIII and IX respectively. The only known wavelength dependency of these uncertainties is in the random errors.
TABLE III

Comparison of the Van den Akker reflectance of $\rho_{v}$ of the walls of two different size spheres coated with $\mathrm{BaSO}_{4}[16]$.

\begin{tabular}{cccc}
\hline \hline \multirow{2}{*}{$\begin{array}{c}\text { Wavelength } \\
(\mathrm{nm})\end{array}$} & \multicolumn{2}{c}{ Sphere radius $\mathrm{R}$} & \\
\cline { 2 - 3 } & $14.6 \mathrm{~cm}$ & $9.5 \mathrm{~cm}$ & \\
\hline 400 & 0.9744 & 0.9744 & 0 \\
425 & .9771 & .9769 & 0.0002 \\
450 & .9789 & .9788 & .0001 \\
475 & .9802 & .9801 & .0001 \\
500 & .9813 & .9812 & .0001 \\
525 & .9823 & .9821 & .0002 \\
550 & $.9829 *$ & .9828 & .0001 \\
575 & .9834 & .9832 & .0002 \\
600 & .9836 & .9835 & .0001 \\
625 & .9838 & .9836 & .0002 \\
650 & .9838 & .9836 & .0002 \\
675 & .9837 & .9836 & .0001 \\
700 & .9836 & .9835 & .0001 \\
725 & .9835 & .9833 & .0002 \\
750 & .9832 & .9831 & .0001 \\
\hline
\end{tabular}

* For this value, $\boldsymbol{\epsilon} \rho_{v}=2.1 \times 10^{-4}$.

TABLE IV

First Determination $\mathrm{BaSO}_{4}[14]$

\begin{tabular}{cccc}
\hline \hline $\begin{array}{c}\text { Wavelength } \\
(\mathrm{nm})\end{array}$ & $\begin{array}{c}\text { Van den Akker } \\
\text { Reflectance } \rho_{v}\end{array}$ & $\rho\left(6^{\circ}, \lambda\right)$ & $F\left(6^{\circ}, \lambda\right)$ \\
\hline 400 & 0.9661 & 0.9606 & 0.9599 \\
425 & .9688 & .9638 & .9631 \\
450 & .9721 & .9675 & .9668 \\
475 & .9744 & .9703 & .9696 \\
500 & .9767 & .9728 & .9721 \\
525 & .9788 & .9752 & .9745 \\
550 & $.9806 \dagger$ & $.9774 *$ & $.9767 @$ \\
575 & .9823 & .9793 & .9786 \\
600 & .9835 & .9807 & .9800 \\
625 & .9845 & .9818 & .9811 \\
650 & .9852 & .9826 & .9819 \\
675 & .9858 & .9834 & .9827 \\
700 & .9864 & .9840 & .9833 \\
725 & .9869 & .9846 & .9839 \\
750 & .9873 & .9850 & .9843 \\
\hline
\end{tabular}

$\dagger \epsilon \rho_{v}$ is $2.3 \times 10^{-4}\left(\delta \rho_{v}=2.1 \times 10^{-4}\right.$ and $\left.\Delta \rho_{v}=1.1 \times 10^{-4}\right)$.

$* \epsilon \rho$ is $9.8 \times 10^{-4}\left(\delta \rho=9.5 \times 10^{-4}\right.$ and $\left.\Delta \rho=2.3 \times 10^{-4}\right)$.

$@ \epsilon \delta$ is $1.01 \times 10^{-3}\left(\delta F=9.5 \times 10^{-4}\right.$ and $\left.\Delta F=3.3 \times 10^{-4}\right)$.

TABLE V

Second Determination Halon [15]

\begin{tabular}{|c|c|c|c|}
\hline $\begin{array}{l}\text { Wavelength } \\
(\mathrm{nm})\end{array}$ & $\begin{array}{l}\text { Van den Akker } \\
\text { Reflectance } \rho_{v}\end{array}$ & $\rho\left(6^{\circ}, \lambda\right)$ & $F\left(6^{\circ}, \lambda\right)$ \\
\hline 400 & 0.9961 & 0.9930 & 0.9927 \\
\hline 425 & .9961 & .9932 & .9929 \\
\hline 450 & .9961 & .9933 & .9930 \\
\hline 475 & .9960 & .9934 & .9931 \\
\hline 500 & .9959 & .9935 & .9932 \\
\hline 525 & .9958 & .9935 & .9932 \\
\hline 550 & $.9957 \dagger$ & $.9935^{*}$ & .9932@ \\
\hline 575 & .9956 & .9935 & .9932 \\
\hline 600 & .9955 & .9935 & .9932 \\
\hline 625 & .9954 & .9935 & .9932 \\
\hline 650 & .9952 & .9934 & .9931 \\
\hline 675 & .9951 & .9934 & .9931 \\
\hline 700 & .9950 & .9933 & .9930 \\
\hline 725 & .9949 & .9932 & .9929 \\
\hline 750 & .9948 & .9931 & .9928 \\
\hline
\end{tabular}


TABLE VI

Third Determination $\mathrm{BaSO}_{4}[16]$

\begin{tabular}{cccc}
\hline $\begin{array}{c}\text { Wavelength } \\
(\mathrm{nm})\end{array}$ & $\begin{array}{c}\text { Van den Akker } \\
\text { Reflectance } \rho_{v}\end{array}$ & $\rho\left(6^{\circ}, \lambda\right)$ & $F\left(6^{\circ}, \lambda\right)$ \\
\hline 400 & 0.9744 & 0.9695 & 0.9688 \\
425 & .9771 & .9732 & .9725 \\
450 & .9789 & .9757 & .9750 \\
475 & .9802 & .9775 & .9768 \\
500 & .9813 & .9791 & .9784 \\
525 & .9823 & .9804 & .9797 \\
550 & $.9829 \dagger$ & $.9813^{*}$ & $.9806 @$ \\
575 & .9834 & .9819 & .9812 \\
600 & .9836 & .9822 & .9815 \\
625 & .9838 & .9824 & .9817 \\
650 & .9838 & .9824 & .9817 \\
675 & .9837 & .9822 & .9815 \\
700 & .9836 & .9819 & .9812 \\
725 & .9835 & .9815 & .9808 \\
750 & .9832 & .9808 & \\
$\dagger \epsilon \rho_{v}$ is $2.3 \times 10^{-4}\left(\delta \rho_{v}=2.1 \times 10^{-4}\right.$ and $\left.\Delta \rho_{v}=1.1 \times 10^{-4}\right)$. \\
$* \epsilon \rho_{v}$ is $2.1 \times 10^{-3}\left(\delta \rho=2.1 \times 10^{-3}\right.$ and $\left.\Delta \rho=2.3 \times 10^{-4}\right)$. \\
$@ \epsilon F$ is $2.1 \times 10^{-3}\left(\delta F=2.1 \times 10^{-3}\right.$ and $\left.\Delta F=3.3 \times 10^{-4}\right)$.
\end{tabular}

The smallest random error is in the central region of the spectrum, where the product of the source intensity and the receiver sensitivity is maximum. At the short wavelength end of the spectrum, there is an increase in uncertainty in $Q_{t}$ and $Q_{s}$ associated with a decrease in source intensity, while at the long wavelength end of the spectrum there is an increase in uncertainty associated with a decrease in receiver sensitivity. However, because the random error associated with $f$ dominates the overall uncertainty, we will cite only error figures at $550 \mathrm{~nm}$ for $\rho_{v}$ as representing the entire spectrum.

The total uncertainty in determining the Van den Akker reflectance is obtained by adding the random and systematic uncertainties in quadrature. The total uncertainty in the Van den Akker reflectance is 0.0002 for the $\mathrm{BaSO}_{4}$ coating. It can be seen that this uncertainty is very small, confirming the results of the error analysis by Goebel, et al., [2]. The larger uncertainty for the Halon coating is due to the transluscence of the coating and is introduced in the extrapolation to the reflectance of a thick enough layer rather than being caused by an uncertainty in the measurement. This additional uncer-

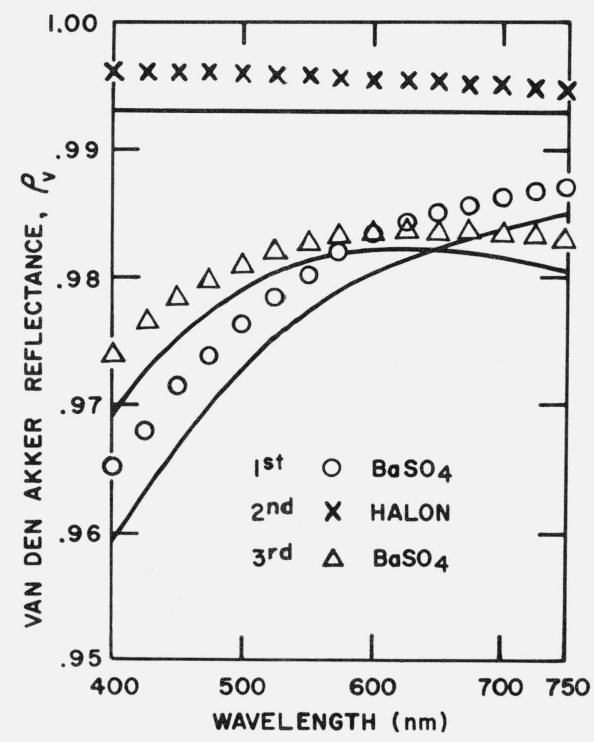

FIGURE 5. Van den Akker reflectance of three coatings.

(The height of the symbols $\times$ represent the total uncertainty. Height of the symbols 0 and $\Delta$ represents approximately 4 times the total uncertainty.) The associated solid curves show $\rho\left(6^{\circ}, \lambda\right)$ for each coating.

tainty would not exist if the Halon coating were twice as thick.

\section{B. Adjustment from Van den Akker Reflectance $\rho_{v}(\lambda)$ to $6^{\circ} / \mathrm{h}$ Reflectance $\rho\left(6^{\circ}, \lambda\right)$}

The second main step is the transition from $\rho_{v}(\lambda)$ to $\rho\left(6^{\circ}\right.$, $\lambda)$. As was pointed out in section III, this step is an intrinsic part of the determination which is needed because the sphere coating is a real material and not an ideal Lambertian reflector. For this reason it is not proper to regard this step as a correction. However, for the type of sphere coatings being examined, the departure from Lambertian reflection is small and the difference between $\rho_{v}$ and $\rho$ is small. For this reason, we will refer to the transition step from $\rho_{v}$ to $\rho$ as an adjustment in order to emphasize the small size of the change with respect to the quantity being changed.

TABLE VII

Typical Values Used in Calculating the Van den Akker Reflectance $\rho_{v}(\lambda=550 \mathrm{~nm})$ for $\mathrm{BaSO}_{4}$ [14].

\begin{tabular}{|c|c|c|c|c|}
\hline \multicolumn{2}{|c|}{ Symbol } & \multirow{2}{*}{$\frac{\text { Reference Equation }}{(16)}$} & \multirow{2}{*}{$\begin{array}{c}\text { Value } \\
12.70 \mathrm{~mm}\end{array}$} & \multirow{2}{*}{$\begin{array}{c}\text { Comments } \\
\text { measured with inside caliper and micrometer. }\end{array}$} \\
\hline & $r$ & & & \\
\hline & $R$ & (16) & $146 \mathrm{~mm}$ & measured with steel rule. \\
\hline & $f$ & (16) & $1.90 \times 10^{-3}$ & calculated using $r$ and $R$ above. \\
\hline & $Q_{s}^{\prime}$ & (19) & $8.731 \times 10^{-2}$ & measured reflectance of auxiliary sphere. \\
\hline & $Q_{p}$ & (19) & $1.24 \times 10^{-3}$ & measured with stainless port open to dark room. \\
\hline & $Q_{f}$ & (19) & $7.0 \times 10^{-1}$ & measured reflectance of stainless steel port plate. \\
\hline & $\omega_{e}$ & (19) & $\begin{array}{c}3.78 \times 10^{-2} \\
\text { radians }\end{array}$ & calculated from dimensions of port structure [17]. \\
\hline & $Q_{s}$ & (19) & $8.630 \times 10^{-2}$ & calculated using $Q_{s}^{\prime}, Q_{p}, Q_{f}$, and $\omega_{e}$ above. \\
\hline & $Q_{t}^{\prime}$ & $(21)$ & $9.832 \times 10^{-1}$ & measured reflectance of target. \\
\hline & $Q_{e}$ & (21) & $1.36 \times 10^{-4}$ & measured with reflectometer port open into dark room. \\
\hline & $Q_{t}$ & $(21)$ & $9.831 \times 10^{-1}$ & calculated using $Q_{t}^{\prime}$ and $Q_{e}$ above. \\
\hline & $\rho_{v}^{\prime}$ & (6) and (A7) & $9.805 \times 10^{-1}$ & $\begin{array}{l}\text { calculated using } f, Q_{s} \text { and } Q_{t} \text { from lines }(\mathrm{c}),(\mathrm{h}) \text { and }(\mathrm{k}) \\
\text { above respectively. }\end{array}$ \\
\hline & & $(26)$ & $25.4 \mathrm{~mm}$ & measured with micrometer caliper. \\
\hline & $W$ & (26) & $0.17 \mathrm{~mm}$ & measured with traveling microscope. \\
\hline & $k_{t}$ & (26) & 1 & $\mathrm{BaSO}_{4}$ \\
\hline & & & (0.9974) & (Halon determination only). \\
\hline & $\rho_{v}$ & (26) & $9.806 \times 10^{-1}$ & calculated using $\rho_{v}^{\prime}, r_{t}, W$, and $k_{t}$ above. \\
\hline
\end{tabular}


TABLE VIII

Calculation of the Random Error in the Van den Akker Reflectance

\begin{tabular}{|c|c|c|c|}
\hline Symbol & Reference Equation & Value & Comments \\
\hline (a) $\delta R$ & (17) & $.8 \mathrm{~mm}$ & $\begin{array}{l}\text { Uncertainty in packing sphere coating to constant ra- } \\
\text { dius. }\end{array}$ \\
\hline (b) $\delta f$ & (17) & $2 \times 10^{-5}$ & Calculated from $\delta R$ above. \\
\hline (c) $\delta Q_{s}^{\prime}$ & $(20)$ & $7 \times 10^{-5}$ & Uncertainty from 3 measurements. \\
\hline (d) $\delta Q_{p}$ & $(20)$ & $7 \times 10^{-5}$ & Roundoff uncertainty. \\
\hline (e) $\delta Q_{s}$ & $(20)$ & $1 \times 10^{-4}$ & Calculated from $\delta Q_{s}^{\prime}$ and $\delta Q_{p}$ as given above. \\
\hline (f) $\delta Q_{t}^{\prime}$ & $(23)$ & $2 \times 10^{-4}$ & See comment on (d) above. \\
\hline (g) $\delta Q_{e}$ & $(23)$ & $7 \times 10^{-5}$ & See comment on (d) above. \\
\hline (h) $\delta Q_{t}$ & $(23)$ & $2.1 \times 10^{-4}$ & Calculated from $\delta Q_{t}^{\prime}$ and $\delta Q_{e}$ above. \\
\hline (i) $\delta \rho_{v}^{\prime}$ & $(25)$ & $\underline{2.1 \times 10^{-4}}$ & $\begin{array}{l}\text { Calculated from } \delta f, \delta Q_{s} \text {, and } \Delta Q_{t} \text { from }(\mathrm{c}),(\mathrm{g}) \text { and }(\mathrm{j}) \\
\text { above. }\end{array}$ \\
\hline (j) $\delta k_{t}$ & $(27)$ & $\left(7 \times 10^{-4}\right)^{*}$ & $\begin{array}{l}\text { Corresponds to an uncertainty in coating thickness of } \\
0.5 \mathrm{~mm} \text {. }\end{array}$ \\
\hline (k) $\delta \rho_{v}$ & $(27)$ & $\begin{array}{l}2.1 \times 10^{-4} \\
\left(7.3 \times 10^{-4}\right)^{*}\end{array}$ & $\begin{array}{l}\text { Calculated from } \delta \rho_{v}^{\prime} \text { and } \\
\delta k_{t} \text { above. }\end{array}$ \\
\hline
\end{tabular}

* Halon coating only.

TABLE IX

Calculation of the Systematic Error in the Van den Akker Reflectance

\begin{tabular}{cccc}
\hline \hline Symbol & Applicable Equation & Value & Comments \\
\hline (a) $\Delta r$ & $(18)$ & $0.03 \mathrm{~mm}$ & $\begin{array}{c}\text { Uncertainty in measuring port } \pm .01 \text { mm. Another } \\
\text { contribution is uncertainty in amount scattered back } \\
\text { from port lip, which is estimated. }\end{array}$ \\
(b) $\Delta f$ & $(18)$ & $9 \times 10^{-6}$ & $\begin{array}{c}\text { Calculated from } \Delta r \text { above. } \\
\text { (c) } \Delta \omega_{e}\end{array}$ \\
(d) $\Delta Q_{s}$ & $(21)$ & $1.1 \times 10^{-4}$ & $\begin{array}{c}\text { Calculated from } \Delta \omega_{e} \text { above and from } Q_{f}, \omega_{e}, \text { and } Q_{s} \\
\text { from entries (f), (g) and (h) in table VII. }\end{array}$ \\
(e) $\Delta \rho_{v}^{\prime}$ & $(25)$ & $9.7 \times 10^{-5}$ & $\begin{array}{c}\text { Calculated from } \Delta f \text { and } \Delta Q_{s} \text { from (b) and (d) above. } \\
\text { (f) } \Delta k_{t}\end{array}$ \\
& $(27)$ & $\left(5 \times 10^{-4}\right)^{*}$ & $\begin{array}{c}\text { Wavelength dependent error } \pm .0005 \text { estimated from } \\
\text { fig. } 4 ; \text { uncertainty due to tapered edges of coating } \\
\pm 0.0001 .\end{array}$ \\
(g) $\frac{r_{t} W}{\left(R^{2}+r^{2}\right)}$ & $(27)$ & $5 \times 10^{-5}$ & $\begin{array}{c}\text { Calculated using } R, r, r_{t}, W \text { from (a), (b), (m), and (n) } \\
\text { in table VII. }\end{array}$ \\
(h) $\Delta \rho_{v}$ & $(27)$ & $\begin{array}{c}\text { Calculated using } \Delta \rho_{v}^{\prime}, \Delta k_{t}, \text { and the gap uncertainty as } \\
\text { given in (e), (f) and (g) above respectively. }\end{array}$
\end{tabular}

* Halon coating only.

\section{Determining the Relative Self-Radiance $\mathbf{N}_{g}(\gamma) / \cos \gamma$}

For each type of sphere coating, the relative self-radiance $N_{g}(\gamma) / \cos \gamma$ was determined as a function of angle of emergence. A $75 \mathrm{~mm}$ radius sphere was coated using the same procedure as was used when coating the spheres for the Van den Akker reflectance determinations. This sphere was irradiated through its single port and the flux emitted from the port past a knife-edge was observed at a number of angles. The geometry of this measurement is illustrated in figure 6.
The instrument used was the NBS-Gaertner goniophotometer illustrated in figure 7 . The source aperture permitted collimation to within $0.25^{\circ}$ of the optical axis, and the receiver was collimated to within $0.6^{\circ}$ of the optic axis. All three coatings were measured with a $V_{\lambda}$-illuminant $C$ [24] spectral weighting, the centroid of which falls at approximately 550 $\mathrm{nm}$. In addition, the coatings from the second and third determinations were measured using a $550 \mathrm{~nm}$ low pass interference filter, which resulted in a centroid of spectral weighting at $450 \mathrm{~nm}$, and using a $600 \mathrm{~nm}$ high pass filter which resulted in a centroid spectral weighting at approxi- 


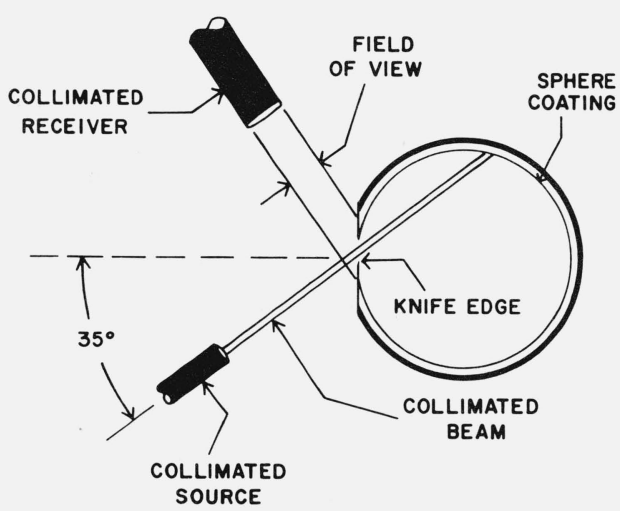

Figure 6. Arrangement of knife-edge apparatus for measuring $N_{g}(\gamma)$.

mately $700 \mathrm{~nm}$. No distinction between the measurements under different spectral weightings were observed, indicating that $L_{s}(\gamma)$ depends principally on the geometrical nature of the surface. The relative magnitude of the observed flux divided by the cosine of the observation angle is shown in figure 8 . Also shown in figure 8 are measurements made by scanning, with a telescopic detector, the interior of a $20 \mathrm{~cm}$ radius sphere coated with Halon. These latter measurements, believed to be much more accurate $( \pm 0.001)$ than the knifeedge measurements, extend only to $45^{\circ}$. Because of the method used to produce the coating by hammering, there is reason to expect an increase in $N_{g}(\gamma) / \cos (\gamma)$ as $\gamma$ approaches $90^{\circ}$ because of a slight glossiness in the surface. However, it appears that this effect is not nearly as great as the measurements using the knife-edge indicate. Two possible sources for the difficulties at large $\gamma$ are reflections from the corner of the knife-edge and scattered light from the receiver optics. At large $\gamma$, the flux to be measured is small, so that small amounts of stray radiation can cause large errors. Fortunately, this uncertainty in $N_{g}(\gamma) / \cos \gamma$ results in only a small uncertainty in determining $C(\Gamma, \lambda)$. A discussion of our

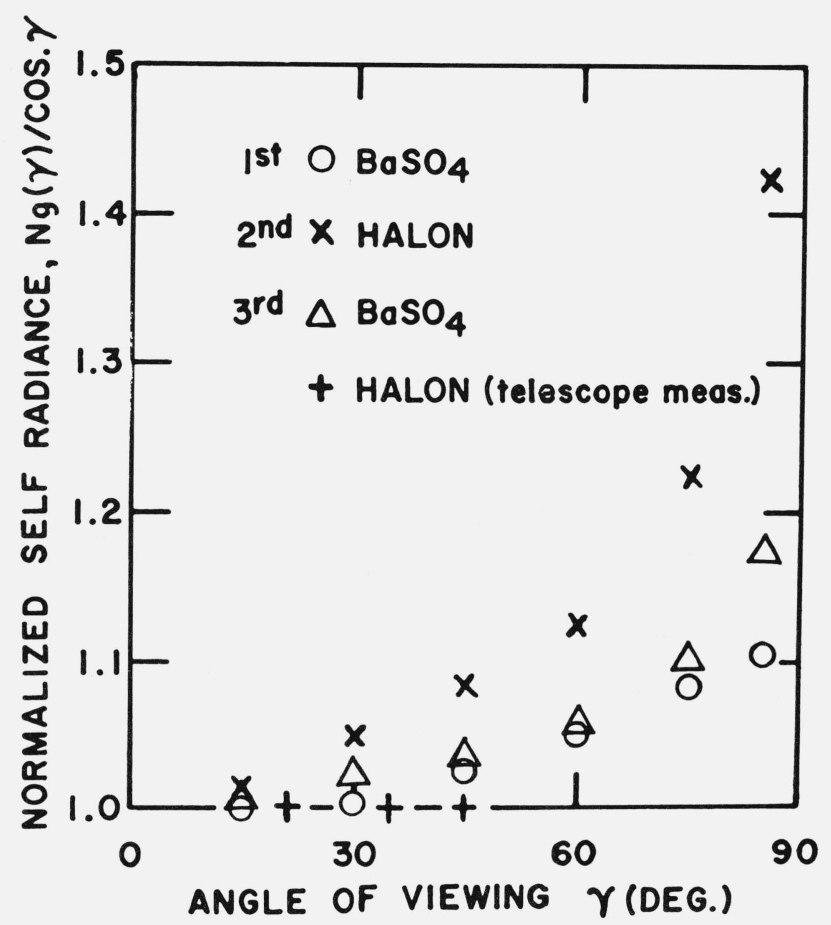

FIGURE 8. Normalized self-radiance at $550 \mathrm{~nm}$ for each of three coating materials.

choice of function to represent $N_{g}(\gamma)$ will be deferred to part IV.B. 3 in which $C(\Gamma, \lambda)$ is calculated.

\section{Determining the Relative Directional-Hemispherical Reflectance $\mathbf{N}_{G}(\Gamma)$}

The relative directional-hemispherical reflectance of a sample of each of the three coatings was measured with the

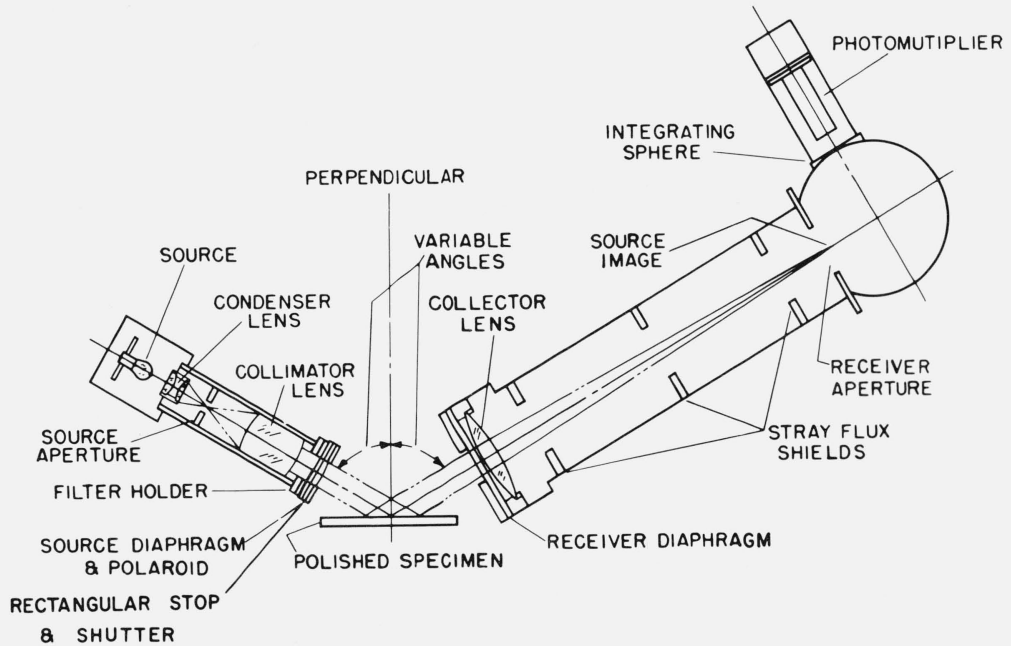

FiguRE 7. NBS-Gaertner goniophotometer. 
DRS using the special measurement accessory apparatus for this purpose [12]. A diagram of this measurement accessory is reproduced from that reference in figure 9. The reflectance values were measured for radiation polarized with the electric vector in the plane of incidence and perpendicular to the plane of incidence. The average of the relative reflectance for the two polarizations was determined as a function of angle of incidence for each material for each of three wavelengths, $450 \mathrm{~nm}, 550 \mathrm{~nm}$, and $750 \mathrm{~nm}$. The data were fitted with a quadratic function, using a least-squares fitting program, to obtain the $N_{G}(\Gamma, \lambda)$ shown in figure 10 . These curves were used to determine the adjustment function $C(\Gamma, \lambda)$ of eq (12). The general trend is for the reflectance to increase as the angle of incidence approaches grazing. The amount of the increase is greater for the materials with lower reflectance.

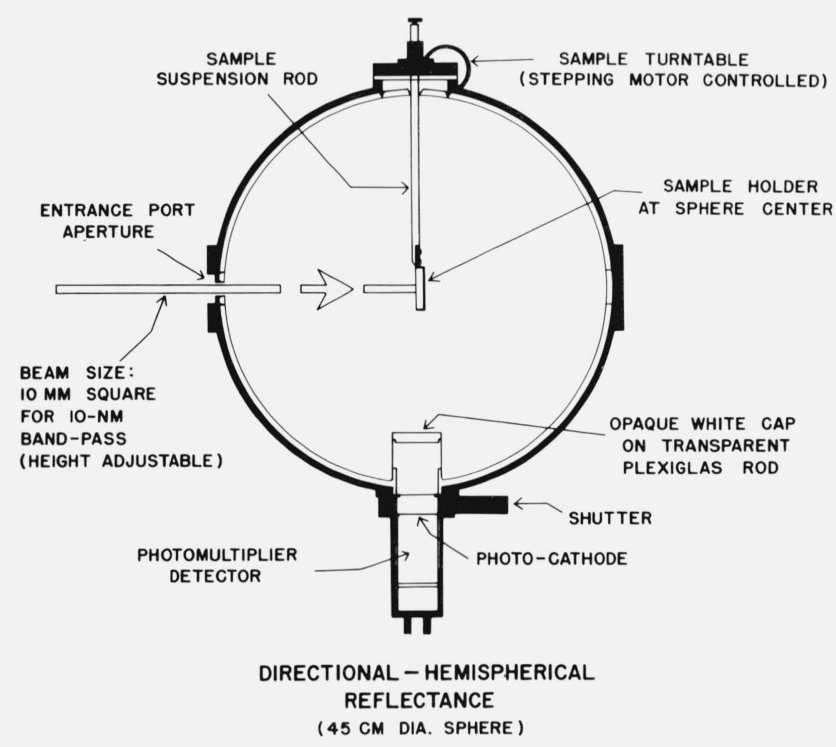

FIGURE 9. Integrating sphere for measuring relative directional-hemispherical reflectance as a function of angle of incidence.

\section{Determining $C(\Gamma, \lambda)$}

For each type of coating, the function $C$ was evaluated using in eq (13) the $N_{g}(\gamma)$ and $N_{G}(\Gamma, \lambda)$ data from parts 1. and 2. above for each of the three wavelengths 450,550 , and 750 $\mathrm{nm}$. Actually three sets of $C$ were calculated for each of three interpretations of the highly uncertain data for $N_{g}(\gamma)$ in figure 8 above in order to evaluate the effect of that uncertainty. We wish to distinguish between the data taken from measurements of the radiance from the sphere wall and the interpretations of this data which are used as incident radiance values in calculating $C(\Gamma, \lambda)$. We will do this by using a lower case $\gamma$ in $N_{g}(\gamma) / \cos \gamma$ to indicate the data obtained directly from the measurements and by using an uppercase $\Gamma$ in $N_{g}(\Gamma) / \cos$ $\Gamma$ to indicate the processed data used as the relative radiance distribution incident on the sphere wall when we calculate $C(\Gamma, \lambda)$. The first set of $C(\Gamma, \lambda)$ was calculated assuming that

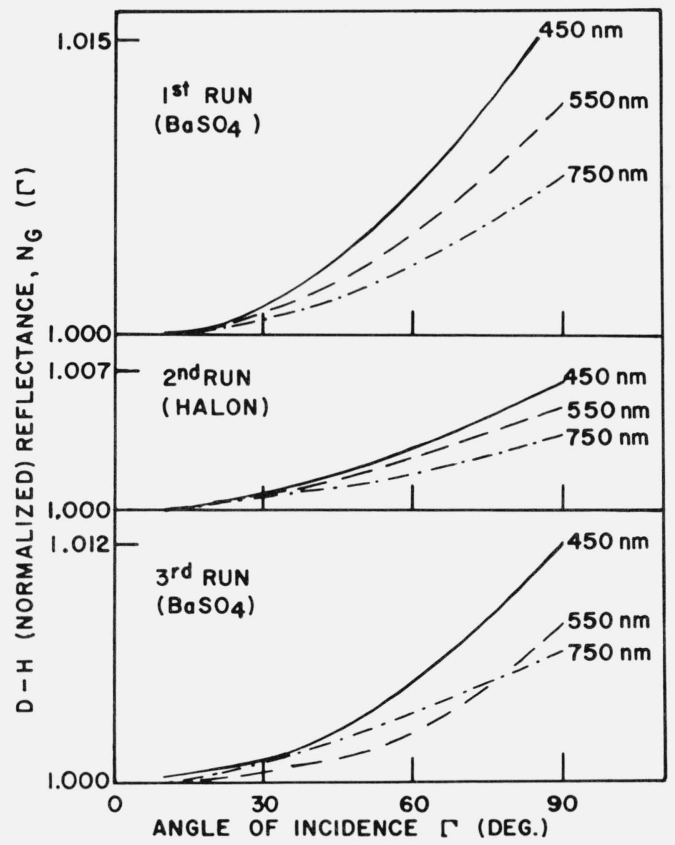

Figure 10. Relative directional-hemispherical reflectance $N_{g}(\Gamma, \lambda)$ as a function of angle of incidence $\Gamma$ for each of three coatings at three wavelengths.

$N_{g}(\gamma) / \cos \gamma$ is independent of $\gamma$. This provides a lower limit on $C$. The second was calculated by using 1 for $N_{g}(\gamma) / \cos \gamma$ between 0 and $45^{\circ}$. To obtain the $N_{g}(\gamma) / \cos \gamma$ above $45^{\circ}$ in this approximation, a straight line was drawn through the knife-edge experiment data from 0 to $45^{\circ}$ and the amount by which the experimental data for greater than $45^{\circ}$ fell above this line was added to 1 to obtain $N_{g}(\gamma)$. (See fig. 11 for a diagram illustrating this procedure for the Halon data in fig. 8.) The set of $N_{g}(\gamma)$ obtained in this way were consistent with the more accurate data from the telescopic detector scan and the $C(\Gamma, \lambda)$ calculated with these $N_{g}(\gamma)$ are used as the accepted values. Finally, as an upper limit, $C$ 's are calculated using the unmodified knife-edge experiment data given in figure 8 . The functions $C(\Gamma, \lambda)$ corresponding to the accepted values, are plotted against $\Gamma$ for each of the three materials at each of three wavelengths in figure 12. (See appendix $\mathrm{C}$ for details of the calculations leading to this figure.)

Since the goal of this section is to determine $\rho\left(6^{\circ}, \lambda\right)$ at 25 $\mathrm{nm}$ intervals over the wavelength range $400 \mathrm{~nm} \leq \lambda \leq 750$ $\mathrm{nm}$, we need values of $C\left(6^{\circ}, \lambda\right)$ at these wavelengths. Since the large amount of data required would make impractical determining all of these values of $C\left(6^{\circ}, \lambda\right)$ in the way described above, we choose to determine $C\left(6^{\circ}, \lambda\right)$ from the data at the three wavelengths 450,550 , and $750 \mathrm{~nm}$ by interpolation and extrapolation. This determination is based on the definition of $C\left(6^{\circ}, \lambda\right)$ which comes from eq (12)

$$
C\left(6^{\circ}, \lambda\right)=\frac{N_{G}\left(6^{\circ}, \lambda\right)}{\bar{N}_{G}}-1
$$




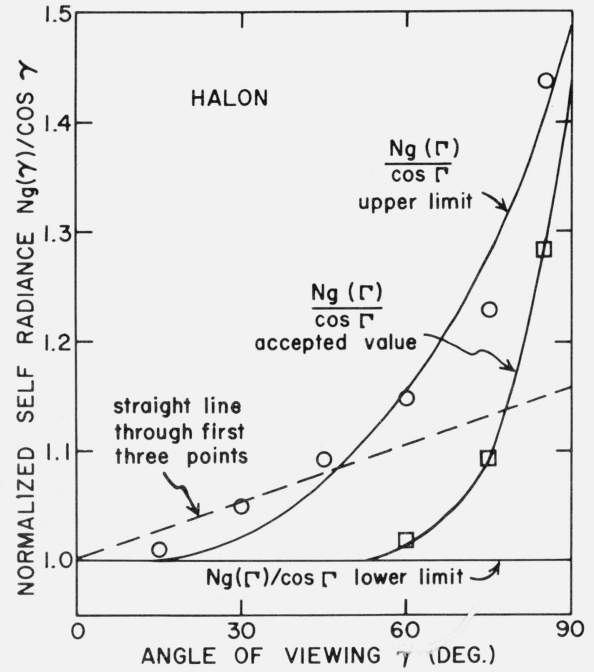

FIGURE 11. An illustration of the three assumptions used in interpreting the $N_{g}(\gamma)$ data from the knife-edge determinations.

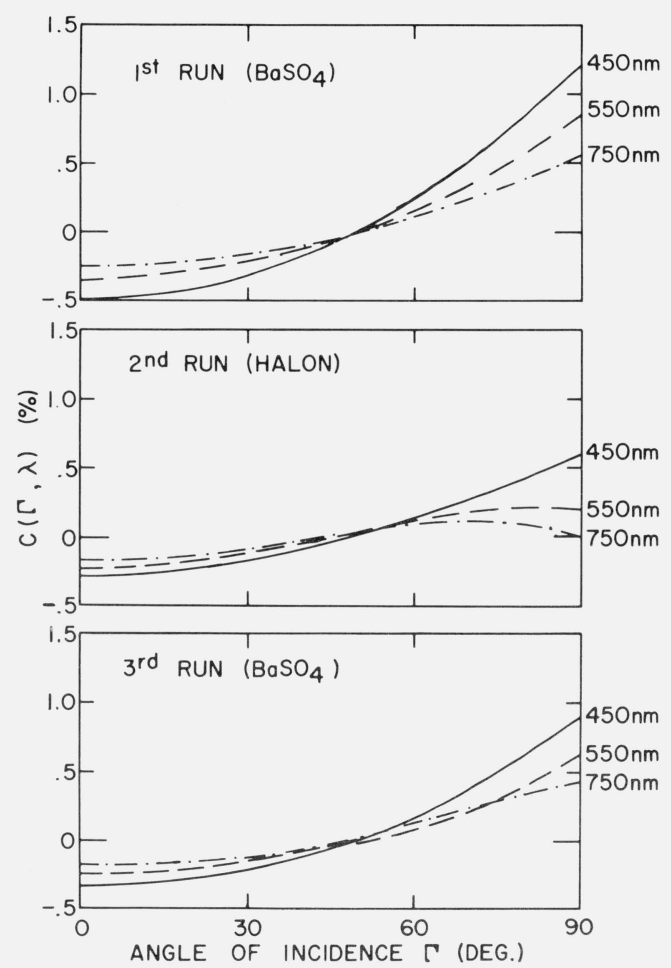

Figure 12. $C(\Gamma, \lambda)$ as a function of $\Gamma$ at three wavelengths for each of three coatings.

Since the weighing to determine $\bar{N}_{G}(\lambda)$ is heaviest at $45^{\circ}$, it follows that

$$
\bar{N}_{G}(\lambda) \simeq N_{G}\left(45^{\circ}, \lambda\right) \text {. }
$$

Making use of this, we can write a correction factor $C^{\prime}\left(6^{\circ}, \lambda\right)$ which is a rough approximation to $C\left(6^{\circ}, \lambda\right)$ as:

$$
C^{\prime}\left(6^{\circ}, \lambda\right)=\frac{N_{G}\left(6^{\circ}, \lambda\right)}{N_{G}\left(45^{\circ}, \lambda\right)}-1
$$

Since $C$ and $C^{\prime}$ are approximately equal, their ratio is a smoothly varying function of wavelength. Therefore, we determined $J(\lambda)$ as

$$
J(\lambda)=d_{0}+d_{1} \lambda+d_{2} \lambda^{2}
$$

such that

$$
J(\lambda)=\frac{C\left(6^{\circ}, \lambda\right)}{C^{\prime}\left(6^{\circ}, \lambda\right)}
$$

at the wavelengths $450 \mathrm{~nm}, 550 \mathrm{~nm}$ and $750 \mathrm{~nm}$. We then determined $C\left(6^{\circ}, \lambda\right)$ at other wavelengths as

$$
C\left(6^{\circ}, \lambda\right)=J(\lambda) C^{\prime}\left(6^{\circ}, \lambda\right)
$$

where $C^{\prime}\left(6^{\circ}, \lambda\right)$ is calculated from expression (32) using values of $N_{G}\left(6^{\circ}, \lambda\right)$ and $N_{G}\left(45^{\circ}, \lambda\right)$ measured with the relative $d / h$ reflectance instrument [12]. The values of $C\left(6^{\circ}, \lambda\right)$ so determined are plotted in fig. 13.

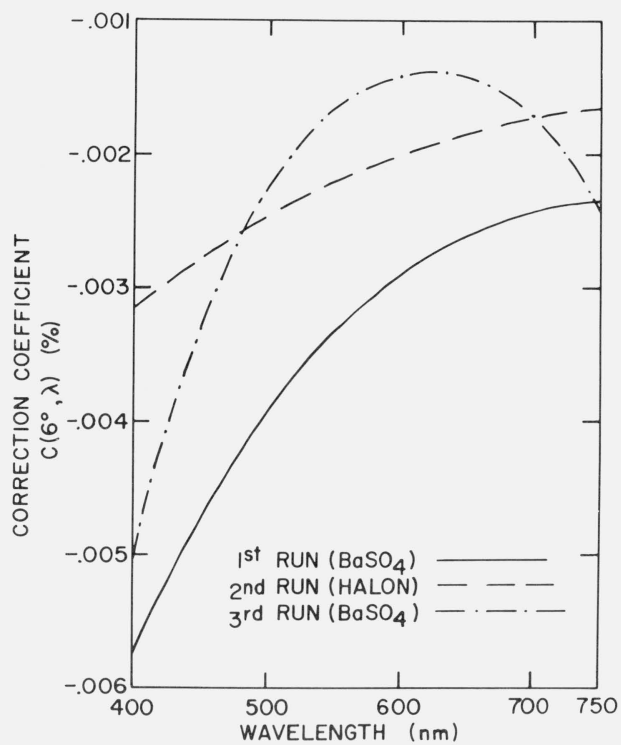

FIGURE 13. $C\left(6^{\circ}, \lambda\right)$ as a function of wavelength for each of three coatings.

\section{Results and Error Analysis}

The three sets of values for $C\left(6^{\circ}, \lambda\right)$ determined above were used in eq (12) to calculate $\rho\left(6^{\circ}, \lambda\right)$ for the three wall materials. The results of this calculation are shown in the third columns of tables IV, V and VI and by the line curves in fig. 5 . 
From eq (12) one can determine a propagation of error formula for the random error $\delta \rho\left(6^{\circ}, \lambda\right)$ as:

$$
\delta \rho\left(6^{\circ}, \lambda\right)=\left[\delta \rho_{v}(\lambda)^{2}+\delta N_{G}\left(6^{\circ}, \lambda\right)^{2}+\delta \bar{N}_{G}(\lambda)^{2}\right]^{1 / 2} .
$$

In this form $\rho_{v}, N_{G}$ and $\bar{N}_{G}$ are each assumed to be 1, and errors common to $N_{G}$ and $N_{G}$ are ignored, since these will effectively cancel when the ratio is taken. Therefore the only errors to be included in eq (36) are the independent errors in the various quantities. The principal independent uncertainty in $\bar{N}_{G}$ is that due to the uncertainty in the self-radiance measurements as shown in appendix $\mathrm{C}$. The principal random uncertainty in $N_{G}$ is due to the noise in the $d / h$ data. Since the value of $N_{G}\left(6^{\circ}, \lambda\right)$ was obtained through a rather indirect procedure involving curve fitting, it is difficult to establish a good theoretical basis for the error estimate. Therefore, we sought a reproducible way of estimating the error which depended as little as possible on arbitrary judgment. Since most of the curves involved fitting through ten to fifteen points with a quadratic function with two independent parameters, there is almost no likelihood that a point taken from the curve will depart from the most probable value by more than the root mean square deviation of the individual points with respect to the curve. Therefore, we will use this deviation as the estimated random uncertainty. In determining $N_{G}$, two such fittings are involved, one to the original data as a function of angle and one to the $C(\lambda)$ data used in the interpolation. A summary of the random error analysis for the $\rho_{v}(\lambda)$ to $\rho\left(6^{\circ}, \lambda\right)$ adjustment is given in table X.

The only source of systematic error which we have identified for this adjustment step is an uncertainty in the measurement of $N_{G}$ due to the correction which is made for the entrance port of the reflectometer. This results in an uncertainty in $C(\Gamma, \lambda)$ of $2 \times 10^{-4}$ independent of wavelength.

The total uncertainty in $\rho\left(6^{\circ}, \lambda\right)$ for a given determination is obtained by adding $\delta \rho\left(6^{\circ}, \lambda\right)$ and $\Delta \rho\left(6^{\circ}, \lambda\right)$ in quadrature.
The additional uncertainty introduced in making the adjustment from $\rho_{v}(\lambda)$ to $\rho\left(6^{\circ}, \lambda\right)$ is of the same order of magnitude as the uncertainty in $\rho_{v}(\lambda)$ itself. Particularly noteworthy is the large increase in uncertainty in the third determination. The barium sulfate used in this determination had a rather "sticky" consistency which made it difficult to pack it into a uniform smooth surface, and this may have had an effect upon the noise in the $N_{G}(\Gamma, \lambda)$ data from this surface.

\section{Adjustment from $d / h$ Reflectance $\rho\left(6^{\circ}, \lambda\right)$ to $d / h$ Reflectance Factor $F\left(6^{\circ}, \lambda\right)$}

In order to determine the bidirectional reflectance factor in the retroreflective direction, apparatus was set up as customarily used for measuring the coefficient of luminous intensity of retroreflective sheeting (See fig. 14). Since the retroreflectance of the sphere coatings is essentially spectrally nonselective over the visible wavelength range [13] we measured the luminous reflectance factor for CIE illuminant $A$ as being representative of the entire spectrum to within the uncertainty of the retroreflectance measurements.

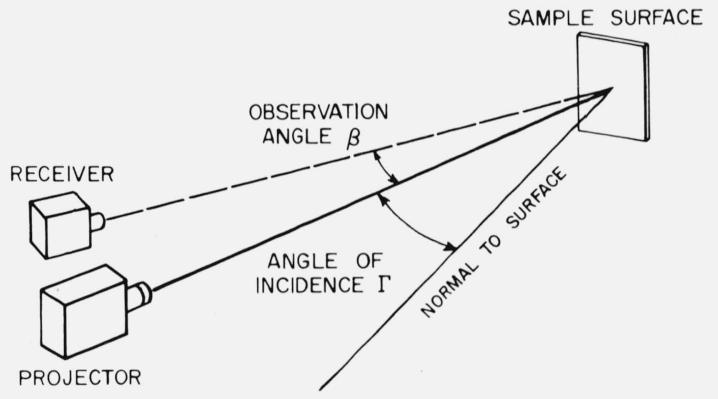

FiguRE 14. Diagram of apparatus used in retroreflectance measurements.

TABLE $X$

Calculation of the errors in the $\boldsymbol{\rho}_{v}(\lambda)$ to $\rho\left(6^{\circ}, \lambda\right)$ adjustment (See equation (36) and the accompanying discussion).

\begin{tabular}{|c|c|c|c|c|c|}
\hline & Symbol & $\mathrm{BaSO}_{4}[14]$ & Halon [15] & $\mathrm{BaSO}_{4}[16]$ & Comments \\
\hline (a) & $\delta \rho_{v}$ & $2.1 \times 10^{-4}$ & $7.3 \times 10^{-4}$ & $2.1 \times 10^{-4}$ & See Table VIII. \\
\hline (b) & $\delta \bar{N}_{G}(\lambda)$ & $0.5 \times 10^{-4}$ & $0.5 \times 10^{-4}$ & $0.5 \times 10^{-4}$ & See Appendix C. \\
\hline (c) & $\delta N_{g}\left(6^{\circ}, \lambda\right)$ & $7 \times 10^{-4}$ & $3 \times 10^{-4}$ & $20 \times 10^{-4}$ & $\begin{array}{l}\text { Component due to fitting angular data } \\
\text { for } N_{G}(\Gamma, \lambda) \text {. }\end{array}$ \\
\hline (d) & $\delta N_{g}\left(6^{\circ}, \lambda\right)$ & $6 \times 10^{-4}$ & $3 \times 10^{-4}$ & $5 \times 10^{-4}$ & $\begin{array}{l}\text { Component due to fitting wavelength } \\
\text { data to } C^{\prime}(\lambda) \text {. }\end{array}$ \\
\hline (e) & $\delta N_{g}\left(6^{\circ}, \lambda\right)$ & $9.2 \times 10^{-4}$ & $4.3 \times 10^{-4}$ & $21 \times 10^{-4}$ & $\begin{array}{l}\text { Quadrature combination of }(\mathrm{c}) \text { and }(\mathrm{d}) \\
\text { above. }\end{array}$ \\
\hline (f) & $\delta \rho\left(6^{\circ}, \lambda\right)$ & $9.5 \times 10^{-4}$ & $7.9 \times 10^{-4}$ & $21 \times 10^{-4}$ & $\begin{array}{l}\text { Quadrature combination of (a), (b) and } \\
\text { (c) above. }\end{array}$ \\
\hline (g) & $\Delta \rho_{v}$ & $1.1 \times 10^{-4}$ & $5.1 \times 10^{-4}$ & $1.1 \times 10^{-4}$ & See Table IX. \\
\hline (h) & $\Delta C$ & $2 \times 10^{-4}$ & $2 \times 10^{-4}$ & $2 \times 10^{-4}$ & $\begin{array}{l}\text { Associated with } d \text { - } h \text { reflectometer port } \\
\text { correction. }\end{array}$ \\
\hline & $\Delta \rho\left(6^{\circ}, \lambda\right)$ & $2.3 \times 10^{-4}$ & $5.5 \times 10^{-4}$ & $2.3 \times 10^{-4}$ & $\begin{array}{l}\text { Quadrature combination of }(\mathrm{g}) \text { and }(\mathrm{h}) \\
\text { above. }\end{array}$ \\
\hline
\end{tabular}


To make the measurements, the receiver was placed in the sample position and a signal $N_{R}$ proportional to the normal illuminance $I_{R}$ on the sample was measured.

$$
N_{R}=k_{R} I_{R}
$$

The receiver was then moved to a position a distance $d$ away from the sample and, on the same scale of measurement and using the same receiver aperture, signals $N_{r}(\beta)$, which is in the same way proportional to the illuminance $I(\beta)$ on the receiver due to the radiation reflected from the sample, was measured with the entire sample area $A$ in view. From the basic definition for reflectance factor, it follows that the reflectance factor $F_{\Gamma}(\beta)$ can be determined from

$$
F_{\Gamma}(\beta)=\frac{N_{r}(\beta)}{N_{R}} \frac{A}{d^{2} \cos (\Gamma) \cos (\Gamma+\beta)} .
$$

The value of $F_{6}(\beta)$ so determined as a function of observation angle $\beta$ is given for $\mathrm{BaSO}_{4}$ and for Halon in fig 15 . (See appendix A, sec. A.2 for further discussion of these data and the evaluation of the integrals.) The retroreflectance factor $F_{6}{ }^{d}(\beta)$ was found to be essentially independent of the angle which the plane of observation makes with respect to the plane of incidence. Therefore, the integral in eq (13) becomes

$\int \omega^{\prime} F(\mathbf{U}, \mathbf{u}) \cos \gamma d \omega=2 \pi \cos 6^{\circ} \int_{0}^{r / d^{\prime}} F_{6}(\beta) \beta d \beta$

and $\omega^{\prime}$ in (13) becomes

$$
\omega^{\prime}=2 \pi \int_{0}^{r / d d^{\prime}} \beta d \beta
$$

where $r$ is the radius of the reflectometer entrance port and $d^{\prime}$ is the distance in the reflectometer sphere from the sample to the plane of the entrance port. The $\rho\left(6^{\circ}, \lambda\right)$ data were adjusted to $F\left(6^{\circ}, \lambda\right)$ using eq (13). In general, the difference $\rho\left(6^{\circ}, \lambda\right)-F\left(6^{\circ}, \lambda\right)$ is 0.0007 for $\mathrm{BaSO}_{4}$ and 0.0003 for Halon. The estimated systematic uncertainty in this adjustment is approximately one-third of its value in each case. These uncertainties, added in quadrature with the uncertainties in $\rho\left(6^{\circ}, \lambda\right)$ yield the total systematic uncertainty in $F\left(6^{\circ}, \lambda\right)$. The values of $F\left(6^{\circ}, \lambda\right)$ for the three coatings are given in the third columns of tables IV, V, and VI.

\section{Determining the $d / h$ Reflectance Factor of Working Standards}

The $d / h$ reflectance factor $F\left(6^{\circ}, \lambda\right)$ determined in IV.C. above is the average for the wall coating used in the Van den Akker sphere. Since this coating is neither permanent nor readily accessible, the reflectance factor $F_{c}\left(6^{\circ}, \lambda\right)$ is determined for working standards made of glass, ceramic, or some other permanent material. The value of $F_{c}$ is assigned as indicated in eq (14) and the accompanying text. Specifically, we will denote by $F_{c, j}\left(6^{\circ}, \lambda\right)$ the reflectance factor for standard $c$ as obtained from the $j$ th determination. Equation (14) takes the form:

$$
F_{c, j}=\frac{Q_{c}}{\bar{Q}_{j}} F_{j}
$$

where

$F_{j} \equiv F_{j}\left(6^{\circ}, \lambda\right)$ is the reflectance factor for the $j$ th wall coating,

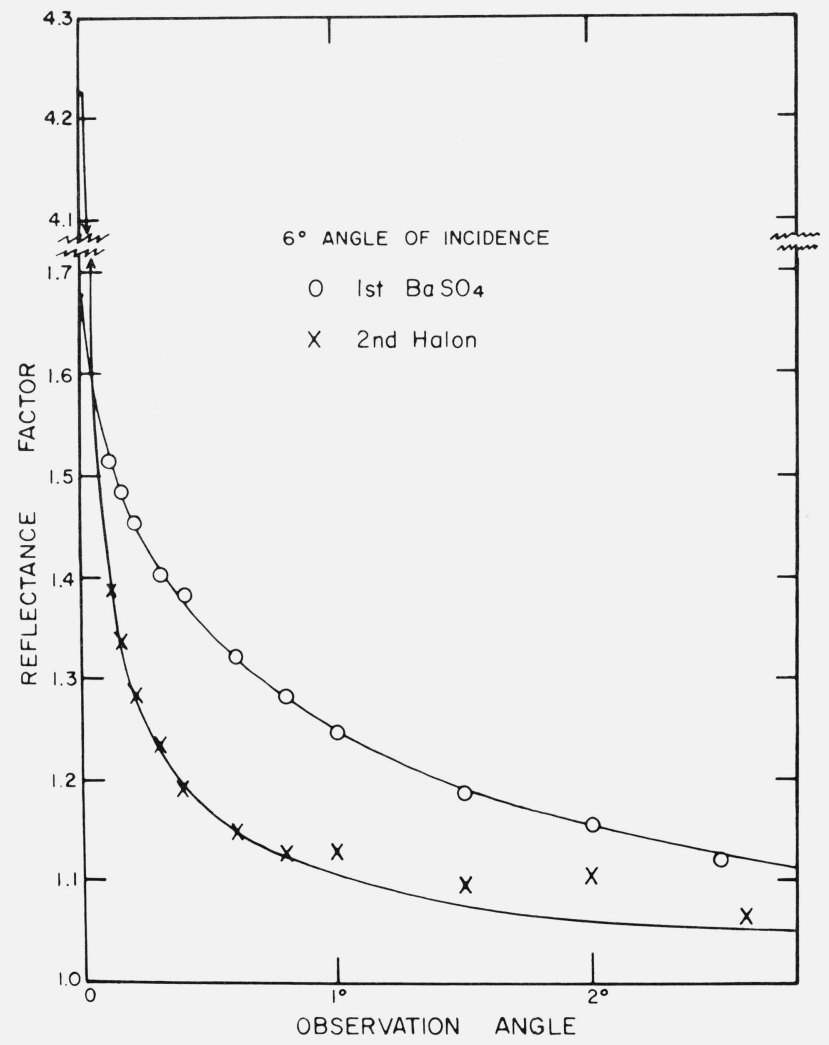

FIGURE 15. Retroreflective bidirectional reflectance factor for $6^{\circ}$ incidence as a function of observation angle.

$Q_{c}$ is the measured relative reflectance of working standard $c$, and

$\bar{Q}_{j}$ is the average of the measured relative reflectance for several representative samples of the $j$ th wall coating.

The relative reflectances $Q_{c}$ and $\bar{Q}_{j}$ were found by experiment to be independent of the polarization of the incident radiation for the working standards and sphere coatings used. It follows that the random error in $F_{c, j}$ is given by:

$$
\delta F_{c, j}=F_{c, j}\left[\left(\frac{\delta Q_{c}}{Q_{c}}\right)^{2}+\left(\frac{\delta \bar{Q}_{j}}{\bar{Q}_{j}}\right)^{2}+\left(\frac{\delta F_{j}}{F_{j}}\right)^{2}\right]^{1 / 2} .
$$

The largest new contribution to the uncertainty which is introduced in this step is $\delta \bar{Q}_{j}$. This is due to the variability between the representative samples and the resulting uncertainty in whether these samples properly represent the sphere coating. If the values of $Q_{c}$ and $Q_{j}$ differ significantly a small correction for instrument non-linearity must be made in the ratio $Q_{c} / \bar{Q}_{j}$. With this non-linearity is associated a small contribution to the systematic uncertainty.

$$
\Delta F_{c, j}=F_{c, j}\left[\left(\frac{\Delta\left(Q_{c} / \bar{Q}_{j}\right)}{\left(Q_{c} / \bar{Q}_{j}\right)}\right)^{2}+\left(\frac{\Delta F_{j}}{F_{j}}\right)^{2}\right]^{1 / 2} .
$$

Three different working standards are currently used. These are identified and described in appendix D. The values of $F_{c, j}$ for each of these standards for each of three determinations are given in tables XI, XII and XIII, and sample calculations for the uncertainties appear in table XIV. 
TABLE XI

Reflectance Factor of Working Standard 1

(Russian Opal Glass, Polished)

\begin{tabular}{|c|c|c|c|c|c|}
\hline $\begin{array}{l}\text { Wavelength } \\
(\mathrm{nm})\end{array}$ & $F_{1,1}$ & $F_{1,2}$ & $F_{1,3}$ & $\bar{F}_{1,2}$ & $\bar{F}_{1,3}$ \\
\hline 400 & 0.9793 & 0.9734 & 0.9738 & 0.9759 & 0.9753 \\
\hline 425 & 0.9781 & 0.9739 & 0.9743 & 0.9756 & 0.9753 \\
\hline 450 & 0.9803 & 0.9765 & 0.9779 & 0.9781 & 0.9781 \\
\hline 475 & 0.9841 & 0.9807 & 0.9812 & 0.9822 & 0.9819 \\
\hline 500 & 0.9854 & 0.9823 & 0.9846 & 0.9836 & 0.9838 \\
\hline 525 & 0.9856 & 0.9821 & 0.9845 & 0.9836 & 0.9838 \\
\hline 550 & 0.9837 & 0.9813 & 0.9830 & 0.9823 & 0.9835 \\
\hline 575 & 0.9810 & 0.9785 & 0.9805 & 0.9796 & 0.9798 \\
\hline 600 & 0.9792 & 0.9761 & 0.9785 & 0.9775 & 0.9777 \\
\hline 625 & 0.9777 & 0.9742 & 0.9769 & 0.9757 & 0.9760 \\
\hline 650 & 0.9770 & 0.9745 & 0.9774 & 0.9756 & 0.9761 \\
\hline 675 & 0.9771 & 0.9751 & 0.9772 & 0.9759 & 0.9763 \\
\hline 700 & 0.9761 & 0.9736 & 0.9761 & 0.9747 & 0.9750 \\
\hline 725 & 0.9742 & 0.9718 & 0.9741 & 0.9728 & 0.9732 \\
\hline 750 & 0.9716 & 0.9687 & 0.9711 & 0.9699 & 0.9702 \\
\hline$\delta$ & $2.2 \times 10^{-3}$ & $1.6 \times 10^{-3}$ & $2.7 \times 10^{-3}$ & $1.3 \times 10^{-3}$ & $1.2 \times 10^{-3}$ \\
\hline$\Delta$ & $3.3 \times 10^{-4}$ & $5.5 \times 10^{-4}$ & $3.3 \times 10^{-4}$ & $5.5 \times 10^{-4}$ & $5.5 \times 10^{-4}$ \\
\hline $\bar{\epsilon}$ & $2.2 \times 10^{-3}$ & $1.7 \times 10^{-3}$ & $2.7 \times 10^{-3}$ & $1.4 \times 10^{-3}$ & $1.3 \times 10^{-3}$ \\
\hline$w$ & 4.5 & 6.0 & 3.7 & - & - \\
\hline$W$ & - & - & - & 10.5 & 14.2 \\
\hline
\end{tabular}

TABLE XII

Reflectance Factor of Working Standard 2 (Vitrolite)

\begin{tabular}{|c|c|c|c|c|c|}
\hline $\begin{array}{l}\text { Wavelength } \\
(\mathrm{nm})\end{array}$ & $F_{2,1}$ & $F_{2,2}$ & $F_{2,3}$ & $\bar{F}_{2,2}$ & $\bar{F}_{2,3}$ \\
\hline 400 & 0.9100 & 0.9049 & 0.9049 & 0.9071 & 0.9065 \\
\hline 425 & .8944 & .8901 & .8907 & .8920 & .8916 \\
\hline 450 & .9006 & .8977 & .8981 & .8990 & .8987 \\
\hline 475 & .9140 & .9116 & .9083 & .9126 & .9114 \\
\hline 500 & .9192 & .9169 & .9178 & .9179 & .9179 \\
\hline 525 & .9242 & .9204 & .9226 & .9221 & .9222 \\
\hline 550 & .9245 & .9222 & .9243 & .9232 & .9235 \\
\hline 575 & .9215 & .9190 & .9213 & .9201 & .9204 \\
\hline 600 & .9160 & .9125 & .9155 & .9140 & .9145 \\
\hline 625 & .9099 & .9066 & .9085 & .9080 & .9082 \\
\hline 650 & .9050 & .9027 & .9043 & .9037 & .9039 \\
\hline 675 & .9035 & .9013 & .9040 & .9023 & .9028 \\
\hline 700 & .9010 & .8991 & .8999 & .8999 & .8999 \\
\hline 725 & .8959 & .8939 & .8953 & .8948 & .8949 \\
\hline 750 & .8893 & .8867 & .8882 & .8878 & .8879 \\
\hline$\delta$ & $2.2 \times 10^{-3}$ & $1.6 \times 10^{-3}$ & $2.3 \times 10^{-3}$ & $1.3 \times 10^{-3}$ & $9.8 \times 10^{-4}$ \\
\hline$\Delta$ & $3.2 \times 10^{-4}$ & $5.3 \times 10^{-4}$ & $3.2 \times 10^{-4}$ & $5.3 \times 10^{-4}$ & $5.3 \times 10^{-4}$ \\
\hline$\epsilon$ & $2.2 \times 10^{-3}$ & $1.7 \times 10^{-3}$ & $2.4 \times 10^{-3}$ & $1.4 \times 10^{-3}$ & $1.1 \times 10^{-3}$ \\
\hline$w$ & 4.5 & 5.9 & 4.2 & - & - \\
\hline$W$ & - & - & - & 10.4 & 14.6 \\
\hline
\end{tabular}

The systematic uncertainty associated with the $F_{c}$ for a particular standard is, by definition, independent of the number of determinations which are made. However, the random uncertainty associated with a given $F_{c}$ can be reduced by taking the average of the $F_{c}$ 's from a number of determinations. We maintain a running weighted average $\bar{F}_{c, j}$ of the results of all determinations from 1 through $j$ by means of the calculation

$$
\bar{F}_{c, j}=\frac{W_{c, j-1} \bar{F}_{c, j-1}+w_{c, j} F_{c, j}}{W_{c, j}}
$$

where the weighting factor $w_{c, j}$ for the $j$ th determination of $F_{c}$ is

$$
w_{c, j}=1 / 100 \epsilon F_{c, j}
$$

where $\boldsymbol{\epsilon} \boldsymbol{F}_{c, j}$ is the total uncertainty in $F_{c, j}$ as is given in appendix $\mathrm{B}$. The weighting factor $W_{c, j}$ for the average of the $j$ determinations is

$$
W_{c, j}=\sum_{i=1}^{j} w_{c, i} .
$$




\section{TABLE XIII}

Reflectance Factor of Working Standard 3

(Porcelain Enamel 80-1)

\begin{tabular}{|c|c|c|c|c|c|}
\hline $\begin{array}{l}\text { Wavelength } \\
(\mathrm{nm})\end{array}$ & $F_{3,1}$ & $F_{3,2}$ & $F_{3,3}$ & $\bar{F}_{3,2}$ & $\bar{F}_{3,3}$ \\
\hline 400 & 0.7615 & 0.7573 & 0.7571 & 0.7591 & 0.7585 \\
\hline 425 & .7936 & .7911 & .7915 & .7922 & .7920 \\
\hline 450 & .8042 & .8019 & .8034 & .8029 & .8030 \\
\hline 475 & .8093 & .8072 & .8073 & .8081 & .8079 \\
\hline 500 & .8105 & .8078 & .8101 & .8090 & .8093 \\
\hline 525 & .8104 & .8091 & .8096 & .8096 & .8096 \\
\hline 550 & .8090 & .8083 & .8094 & .8086 & .8088 \\
\hline 575 & .8063 & .8045 & .8071 & .8053 & .8058 \\
\hline 600 & .8030 & .8017 & .8032 & .8022 & .8025 \\
\hline 625 & .7990 & .7969 & .7994 & .7978 & .7983 \\
\hline 650 & .7949 & .7937 & .7960 & .7942 & .7947 \\
\hline 675 & .7926 & .7911 & .7925 & .7917 & .7919 \\
\hline 700 & .7909 & .7900 & .7927 & .7904 & .7910 \\
\hline 725 & .7885 & .7869 & .7886 & .7875 & .7878 \\
\hline 750 & .7855 & .7839 & .7860 & .7846 & .7850 \\
\hline$\delta$ & $2.1 \times 10^{-3}$ & $1.5 \times 10^{-3}$ & $2.3 \times 10^{-3}$ & $1.3 \times 10^{-3}$ & $1.1 \times 10^{-3}$ \\
\hline$\Delta$ & $3.0 \times 10^{-4}$ & $4.7 \times 10^{-4}$ & $3.0 \times 10^{-4}$ & $4.7 \times 10^{-4}$ & $4.7 \times 10^{-4}$ \\
\hline$\epsilon$ & $2.2 \times 10^{-3}$ & $1.6 \times 10^{-3}$ & $2.3 \times 10^{-3}$ & $1.4 \times 10^{-3}$ & $1.2 \times 10^{-3}$ \\
\hline$w$ & 4.6 & & 4.2 & - & - \\
\hline$W$ & - & - & - & 10.8 & 15.0 \\
\hline
\end{tabular}

TABLE XIV

Calculation of the Uncertainties in the $\rho\left(6^{\circ}, \lambda\right)$ to $F\left(6^{\circ}, \lambda\right)$ and $F\left(6^{\circ}, \lambda\right)$ to $F_{c, j}\left(6^{\circ}, \lambda\right)$ steps as determined for Working Standard No. 2 .

\begin{tabular}{|c|c|c|c|c|c|}
\hline & \multirow{2}{*}{ Symbol } & \multicolumn{3}{|c|}{ Value } & \multirow{2}{*}{ Comments } \\
\hline & & $\mathrm{BaSO}_{4}[14]$ & Halon [15] & $\mathrm{BaSO}_{4}[16]$ & \\
\hline (a) & $\begin{array}{l}\delta F\left(6^{\circ}, \lambda\right) \\
\quad\left(\simeq \delta \rho\left(6^{\circ}, \lambda\right)\right)\end{array}$ & $9.5 \times 10^{-4}$ & $7.9 \times 10^{-4}$ & $2.1 \times 10^{-3}$ & See table X, entry (f). \\
\hline (b) & $F_{j}$ & 0.9767 & 0.9932 & 0.9806 & See tables IV, V, and VI. \\
\hline (c) & $\delta Q_{c}$ & $2.7 \times 10^{-4}$ & $8.7 \times 10^{-4}$ & $3 \times 10^{-4}$ & $\begin{array}{l}\text { From three measurements in each determina- } \\
\text { tion. }\end{array}$ \\
\hline (d) & $Q_{c}$ & 0.929833 & 0.931036 & 0.92922 & Measured. \\
\hline (e) & $\delta \bar{Q}_{j}$ & $2 \times 10^{-3}$ & $1.2 \times 10^{-3}$ & $1.2 \times 10^{-3}$ & From measurements on a number of samples. \\
\hline $\begin{array}{l}(f) \\
(\mathrm{g})\end{array}$ & $\begin{array}{l}\bar{Q}_{j} \\
F_{2, j}\end{array}$ & $\begin{array}{l}0.985022 \\
.9245\end{array}$ & $\begin{array}{l}1.00283 \\
0.9222\end{array}$ & $\begin{array}{l}0.985982 \\
0.9243\end{array}$ & $\begin{array}{l}\text { Measured. } \\
\text { Calculated from (b), (d) and (f) above in equa- } \\
\text { tion (41). }\end{array}$ \\
\hline (h) & $\delta F_{2, j}$ & $2.2 \times 10^{-3}$ & $1.6 \times 10^{-3}$ & $2.3 \times 10^{-3}$ & $\begin{array}{l}\text { Calculated from equation (42) and (a) through } \\
\text { (g) above. }\end{array}$ \\
\hline (i) & 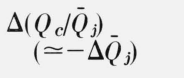 & $-1 \times 10^{-4}$ & $-1 \times 10^{-4}$ & $-1 \times 10^{-4}$ & From reference [17]. \\
\hline (j) & $\Delta F_{j}$ & $3.3 \times 10^{-4}$ & $5.6 \times 10^{-4}$ & $3.3 \times 10^{-4}$ & From tables IV, V, and VI. \\
\hline (k) & $\Delta F_{2, j}$ & $3.2 \times 10^{-4}$ & $5.3 \times 10^{-4}$ & $3.2 \times 10^{-4}$ & $\begin{array}{l}\text { Calculate from equation (43) and (b), (d), (f), } \\
\text { (i) and (j) above. }\end{array}$ \\
\hline (l) & $\epsilon F_{2, j}$ & $2.2 \times 10^{-3}$ & $1.7 \times 10^{-3}$ & $2.4 \times 10^{-3}$ & Quadrature combination of (i) and ( $k$ ) above. \\
\hline (m) & $w_{c, j}$ & 4.5 & 5.9 & 4.2 & Calculate from equation (45) using (1) above. \\
\hline (n) & $W_{c, j-1}$ & - & 10.4 & 14.6 & Calculate from equation (46) using $(\mathrm{m})$ above. \\
\hline (o) & $\bar{F}_{c, j}$ & - & 0.9232 & 0.9235 & $\begin{array}{l}\text { Calculate from equation (44) using }(\mathrm{g}) \text { and }(\mathrm{m}) \\
\text { above. }\end{array}$ \\
\hline (p) & $\delta \bar{F}_{c, j}$ & - & $1.3 \times 10^{-3}$ & $9.8 \times 10^{-4}$ & $\begin{array}{l}\text { Calculate from equation (47) using }(\mathrm{g}),(\mathrm{m}) \text {, } \\
(\mathrm{n}) \text {, and (o) above. }\end{array}$ \\
\hline (q) & $\Delta \bar{F}_{c, j}$ & - & $5.3 \times 10^{-4}$ & $5.3 \times 10^{-4}$ & Largest entry from (k) above. \\
\hline (r) & $\epsilon F_{c, j}$ & - & $1.4 \times 10^{-3}$ & $1.1 \times 10^{-3}$ & Quadrature combination of $(p)$ and $(q)$ above. \\
\hline
\end{tabular}


The random uncertainty in the $\bar{F}_{c, j}$ is given by

$$
\delta \bar{F}_{c, j}=\frac{\left[\left(W_{c, j-1} \delta \bar{F}_{c, j-1}\right)^{2}+\left(w_{c, j} \delta F_{c, j}\right)^{2}\right]^{1 / 2}}{W_{c, j}} .
$$

The values of $\bar{F}_{c, 2}$ and $\bar{F}_{c, 3}$ appear in tables XI, XII, and XIII along with the $F_{c, j}$. The results of these three determinations are also displayed by way of summary in figure 16.

\section{Conclusions}

\section{A. Precautions and Pertinent Parameters}

In the work reported in this paper, a large number of potential sources of error in determining reflectance factor using the Van den Akker method were investigated in order to evaluate the magnitude of their importance. Many of these sources of uncertainty were of little importance in themselves and even cumulatively were of importance only to a laboratory seeking the highest accuracy possible. For a laboratory for which a total uncertainty on the order of \pm 0.005 is tolerable, a number of the detailed steps described in section IV are not necessary. In this section we will briefly describe precautions which should still be taken in the case of such truncated measurement procedures and make suggestions for possible improvements in the overall procedure.

\section{Determining the Van den Akker Reflectance $\rho_{v}(\lambda)$}

In an experiment designed and carried out with reasonable care, the Van den Akker reflectance $\rho_{v}$ can be determined very accurately. The principal strong point in this method is the insensitivity of the basic measurement accuracy to uncertainties in any of the measured parameters such as the port diameter, the sphere diameter, or the ratio of the reflectance of the sphere to the reflectance of the wall sample. The sensitivity to such parameters is kept lowest by using a large sphere with a small port (low $f$ ) and by using a highly reflecting coating in the sphere so that the sphere reflectance is still reasonably high.

Considerable care should be taken however to make the sphere coating in such a way that the $\mathrm{V}$ an den Akker reflectance can be related meaningfully to the reflectance of a sample of the coating. This means that a coating should be chosen the reflectance of which is uniform and very reproducible. It is especially important that no cracks appear in the finished coatings and that the coating should be thick enough to be opaque. In this regard, it is probably best not to incorporate a removable port in the $\mathrm{V}$ an den Akker sphere at all, but rather to rely upon the reproducibility of the coating and use the average $Q$ from several separate samples of coating for $Q_{t}$ in eq (6).

\section{Adjustment from the Van den Akker Reflectance $\boldsymbol{\rho}_{v}$ to the $\mathrm{d} / \mathrm{h}$ Reflectance $\rho\left(6^{\circ}, \lambda\right)$}

As can be seen from figure 13, the magnitude of this adjustment was in no case greater than -0.006 . Since $\rho(\Gamma$, $\lambda)$ can never be greater than 1 , it follows that this adjustment will be smaller for samples for which $\rho_{v}$ closer to 1 . It also is clear that the need for this adjustment comes about from the rise in reflectance at near-grazing incidence. This rise is due to the specular reflectance of the slightly glossy surface which is produced in pressing. For this reason, a coating technique which produces a rougher or more matted coating is to be preferred if no adjustment is to be made. It is probably the roughness of the coating that Goebel, et al., [2] produced by scraping which led to the rather remarkably good agreement between their results, which are the basis of the earlier NBS scale of $F\left(6^{\circ}, \lambda\right)$, and the present adjusted results. (See fig. 17 and the discussion in section V.C. which follows.) An error of as much as 0.01 in the upward direction can result from assuming $\rho_{v}$ and $\rho$ to be equal, and an adjustment based on relative $d / h$ reflectance measurements $N_{G}(\Gamma, \lambda)$ should be made if an uncertainty of less than \pm .01 is desired. In all but the highest accuracy work, it is safe to assume the selfradiance is Lambertian, i.e., $N_{g}(\gamma, \lambda) / \cos \gamma$ is constant.

It should be pointed out that in every case $\rho_{v}$ and $\rho_{45 \% h}$ are very nearly equal, as can be determined from the $C(\Gamma, \lambda)$ curves in figure 12. Therefore the $\mathrm{V}$ an den Akker method is especially well adapted for calibrating measurements of directional-hemispherical reflectance at $45^{\circ}$ incidence.

\section{Adjustment from $d / h$ Reflectance $\rho\left(6^{\circ}, \lambda\right)$ to $d / h$ Reflectance Factor $F\left(6^{\circ}, \lambda\right)$}

Since most reflectometers compare reflectance factor $F\left(6^{\circ}\right.$, $\lambda)$ rather than reflectance $\rho\left(6^{\circ}, \lambda\right)$, it is in principle necessary to make an adjustment. However, since the solid angle subtended at the sample is small compared to the total hemisphere, any departure from Lambertian reflectance by the sphere coating would have to be large if the difference between $\rho$ and $F$ is to be significant. In the sphere coating materials ordinarily in use, the reflectance factor departs greatly from 1 only for small solid angles of collection at very small observation angles $\beta$ (fig. 14). Therefore in general $\rho$ is less than 0.001 higher than $F$ and this adjustment can be ignored or estimated from data in the literature [13].

Note that if the object of the measurement is to determine the radiative transfer properties of the sphere coating itself, then $\rho$ is the quantity of interest and the adjustment to $F$ should not be made. This would be the case, for example, if the coating were being studied in order to determine the radiative heat transfer to the coating material.

\section{Determining the $d / h$ Reflectance Factor $F\left(6^{\circ}, \lambda\right)$ of Working Standards}

Next to the adjustment from $\rho_{v}$ to $\rho$, it is this step which contains the highest potential for error. These measurements are meaningful only to the extent that the samples of coating with which the working standards are compared are representative of the sphere wall coating. It is for this reason that the sphere coating and the samples should be prepared in precisely the same way and that reproducibility of reflectance is an important requirement of the coating. It is also this reason that favors Van den Akker's original technique of having many removable sections in the sphere wall itself, provided that the presence of such removable sections does not give rise to irregularities in the sphere coating.

\section{B. The Present NBS Scale of $d / h$ Reflectance Factor $F\left(6^{\circ}, \lambda\right)$}

The present NBS scale of spectral $d / h$ reflectance factor is maintained by means of three carefully preserved working standards which have been evaluated at $25 \mathrm{~nm}$ intervals. The reflectance factor data for the first three determinations are given in tables XI, XII and XIII. Figure 16 provides a 


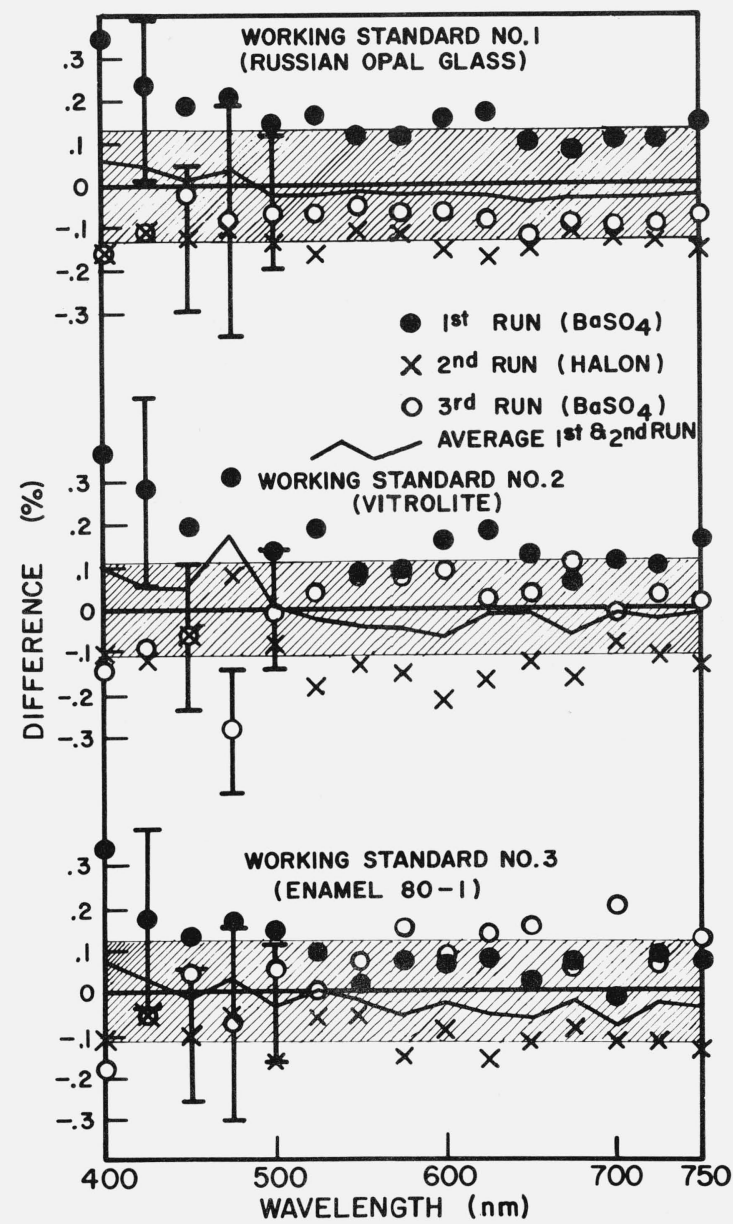

FigurE 16. Reflectance factor differences for the measurement of three working standards in three reflectance scale determinations.

graphical record of the establishment of the average value for the three determinations. The representative error bars given to the left of the diagram actually were derived for $550 \mathrm{~nm}$, but are fairly representative of the entire spectral range. The zero line in each case represents the average for the three determinations, and the shaded area represents the range of the total uncertainty of that average. Although the distribution of the points in general is consistent with the uncertainty levels which have been assigned, there is a lack of consistency from standard to standard between the relative values from pairs of determinations. For example, for working standard No. 1 (Russian opal glass), the third determination reflectance values are consistently lower than those of the first determination, whereas, for the other two working standards the first and third determinations yielded values which were more nearly equal (fig. 16). This difference in general behavior is too large to be explained by the uncertainties in the measured values of $Q_{c}$ in expression (41). The most straightforward explanation for this difference is a slight drop in the reflectance of the working standard No. 1 during the interval between the second and third determinations. A better knowledge of the stability of the working standards will be obtained as more data are gathered from additional determinations.

The results of the work reported in this paper document the establishment of a scale of $d / h$ reflectance factor at $25 \mathrm{~nm}$ intervals over the wavelength range 400 to $750 \mathrm{~nm}$. The error analysis indicates that for high quality, uniform samples with reflectance greater than 0.5 there is only a very small probability that our measured reflectance factor values will be in error by more than 0.0015 .

\section{Relationship to Other Scales}

In order to determine the relationship between the newly established NBS scale of $d / h$ reflectance factor and the former one, we measured the three working standards on the NBS scale established in 1965. The results of this comparison are summarized in figure 17. In this figure, the data points indicate the departure of the old scale from the new for each working standard. The departure of the average value of the spectral $6^{\circ} / h$ reflectance factors for the three standards on the 1965 scale from the same average on the new scale is shown by the light continuous line (GE average). The shaded area in the figure represents the uncertainty of the new scale, and the error bar on a central point indicates the uncertainty attributed to the 1965 scale. On the average, the old scale appears to depart from the new by about 0.002 at the short wavelength end of the range with the magnitude of the departure diminishing as the wavelength increases.

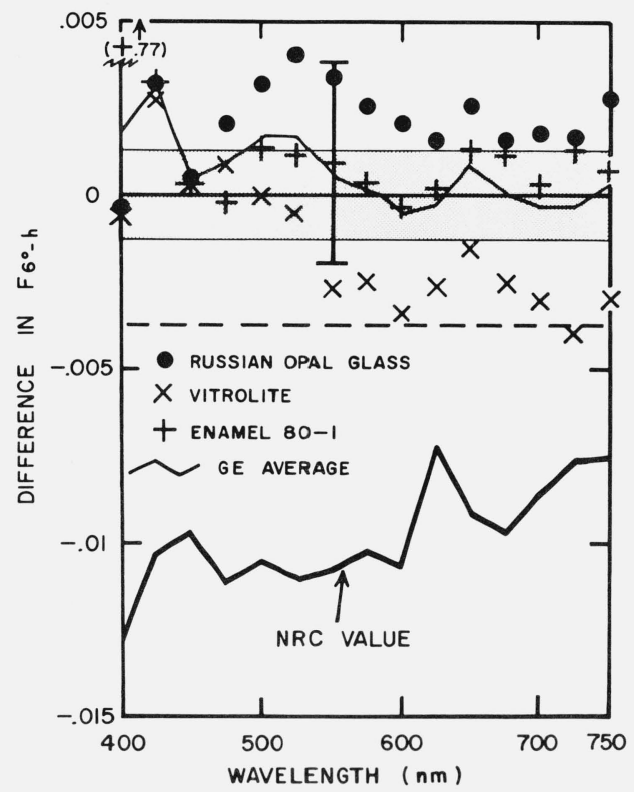

FIGURE 17. Comparison of present NBS scale of $F_{6^{\circ}-h}$ (taken as zero line) to previous NBS scale and to Results of an intercomparison with NRC.

In 1974, measurements were made of several samples of pressed $\mathrm{BaSO}_{4}$ and matte ground Russian opal glass at the National Research Council Laboratories (NRC) in Canada and at NBS on the 1965 NBS scale. The heavy line in figure 17 (NRC value) departs from the light line (GE average) by the difference between the averages of the reflectance values determined in the intercomparison. In this way, the present NBS scale and the NRC scale of 1974 are compared indirectly. The rather noisy appearance of the difference can very 
probably be attributed to the imprecision of the NBS GE spectrophotometer.

The dashed line at approximately -0.004 indicates the combined error for this comparison. Since the NRC value differs from the present NBS value by over twice that amount, it is clear that at least one of these scales is in error by an amount greater than that claimed for it. At the time of this writing, work is under way on a new direct intercomparison between the present NRC and NBS scales of directionalhemispherical reflectance factor and on joint efforts to determine the cause of any differences.

\section{Looking Ahead}

We have already begun work on a determination of $d / h$ reflectance factor by the Sharp-Little method [25, 26]. Most of the data have been obtained, and detailed analysis will be begun soon. Preliminary results indicate that the reflectance factors of the three working standards obtained by that method agree with those obtained by the Van den Akker method to well within the uncertainty of 0.0015 . Therefore, it appears that the present NBS scale is accurate to within the uncertainty reported in this paper.

Mainly because of the slight instability and non-uniformity of the working standards, it is doubtful that an uncertainty in $F\left(6^{\circ}, \lambda\right)$ of less than 0.1 percent of its value can be achieved on a practical, routine basis by any technique which relies on such standards. However, these working standards are more stable and more uniform than most samples encountered in practice. For this reason, we believe the newly established NBS scale of $d / h$ reflectance factor to be sufficiently accurate and precise to serve the current needs for such measurements in science and industry.

\section{References}

[1] Venable, William H. Jr., The National Measurement System for Spectrophotometry (Part of NBS Special Publication series 445, currently being prepared for publication.)

[2] Goebel, David G., Caldwell, B. Patrick, Hammond, Harry K., III, Use of an auxiliary sphere with a spectrophotometer to obtain absolute reflectance, J. Opt. Soc. Amer. 56, 783 (1966).

[3] Certain products are mentioned in this paper by brand name or manufacturer for purposes of identification. This in no way implies recommendation or endorsement by the National Bureau of Standards.

[4] Keegan, Harry J., and Gibson, Kasson S., On the use of working standards of didymium and Vitrolite glasses for spectrophotometric measurements. J. Opt. Soc. Amer. 34, 770 (Dec. 1944). (abstract).

[5] J. Opt. Soc. Amer. 28 (Oct. 1938). (Issue devoted to early development of recording spectrophotometer).

[6] NBS Letter Circular 1050 (June 1970). Preparation and Colorimetric Properties of a Magnesium-Oxide Relectance Standard.

[7] Private communication from W. Erb., Physikalisch-Technische Bundesanstalt.

[8] Venable, William H. Jr., Hsia, Jack J., and Weidner, Victor R., Development of an NBS reference spectrophotometer for diffuse reflectance and transmitance, Nat. Bur. Stand. (U.S.), Tech. Note 594-11, 47 pages (Oct. 1976).

[9] Van den Akker, J. A., Dearth, L. R., and Shillcox, W. M., Evaluation of absolute reflectance for standardization purposes, J. Opt. Soc. Am. 56, 250 (1966).

[10] Venable, William H. Jr., and Hsia, Jack J., Describing spectrophotometric measurements, Nat. Bur. Stand. (U.S.), Tech. Note 594-9, 50 pages (Nov. 1974).

[11] International Lighting Vocabulary, Publication CIE No. 17 (E-1.1) 1970 , section 45-20-201.

[12] Section III.B. of reference [8] above.

[13] Egan, W. G., and Hilgeman, T., Retroreflectance measurements of photometric standards and coatings, Applied Optics 15, 1845 (1976).
[14] $\mathrm{BaSO}_{4}$ manufactured by Mallincrodt Chemical Works (Reagent powder for X-ray diagnosis) \#3808. [3]

[15] Halon tetrafluoroethylene powder manufactured by Allied Chemical Co. Type G-80, Lot 463169 Drum 31. [3]

[16] $\mathrm{BaSO}_{4}$ manufactured by J. T. Baker Chemical Co. \#5-1030 (5531011) (analytical Reagent) [3].

[17] See section III. A. of reference [8] above.

[18] See Appendix C of Reference [8] above.

[19] Jenkins, F. A., and White, H. E., Fundamentals of Optics, 3rd edition (McGraw-Hill, N. Y., 1957) pg. 521, figure 25M.

[20] Jakob, Max, Heat Transfer, Volume II, p. 14 (John Wiley and Sons, N. Y., 1963), $\left(\omega_{e} / \pi=1-f_{12}\right)$.

[21] See section III.A.4 of reference [8] above.

[22] Kubelka, Paul, New contributions to the Optics of Intensely LightScattering Materials. Part I, J. Opt. Soc. Amer. 38, 448 (1948).

[23] Grum, F. and Saltzman, M.; P-75-77 New White Standard of Reflectance. Compte Rendu $18^{\mathrm{e}}$ Session, Londres 1975, CIE Publication No. 36, 91 (1976).

[24] Colorimetry, Publication CIE No. 15 (E13.1) 1971, pages 81 and 93.

[25] Sharp, C. H. and Little, W. F., Measurement of Reflection Factors, Transactions I.E.S. 15, $802(1920)$

[26] Budde, Wolfgang, and Dodd, C. X., Absolute reflectance measurements in the $D / 0^{\circ}$ Geometry, Die Farbe 19, 94 (1970).

[27] Hapke, Bruce W., A theoretical photometric function for the lunar surface, Journal of Geophysical Research 68, 4571 (1963).

[28] Born, M., and Wolf, E., Principles of Optics, 3rd Edition, (Pergamon Press, New York, 1964), p. 399.

\section{Appendix A. Accounting for Retroreflectance}

Retroreflectance refers to the tendency of objects to preferentially reflect radiation back in the direction from which it is incident. This can arise from several causes. If a focussing element such as a small dielectric sphere directs radiation onto a reflecting surface at or near its focal point, the reflected radiation will be returned back into the direction from which it came. This type of retroreflectance can be very directional, i.e., most of the radiation returns within a few tenths of a degree of the angle from which it came. A second, much less directional retroreflectance effect, results from the fact that shadows are not visible from the direction of incidence. This mechanism is important only for surfaces which are not highly reflecting, so that the radiation is not rereflected out of the shadows. In the case of the highly reflecting coatings being used in the spheres in this experiment, the highly directional retroreflectance dominates.

\section{The Effect of Retroreflectance}

In order to obtain an estimate of how the presence of retroreflectance will affect a determination of the $\mathrm{V}$ an den Akker reflectance $\rho_{v}$, we will use a simple model in which most of the radiation is reflected in a Lambertian distribution but a small retroreflected fraction $f_{r}$ appears above the Lambertain background. The effect which the retroreflectance has upon the flux $\phi_{i}(\lambda)$ (see III.A.) striking the sphere boundary can be seen by going one step backwards in determining the source of $\phi_{i}$. The flux $\phi_{i}$ comes from the walls by reflection, and the incident radiation for this reflection is from the sphere walls, but not from the port. Therefore, in the presence of retroreflection the irradiance on the sphere boundary is higher on the wall coating than in the port. The makeup of $\phi_{i}$ can be determined by noting that, in this model, the flux incident upon the walls (which also comes from the walls) is either absorbed or reflected. The total reflected flux, which is $\phi_{i}$, is made up of two parts

$$
\phi_{i}=\phi_{r}+\phi_{i}^{\prime}
$$


where $\phi_{r}$ is the retroreflected fraction of the reflected flux

$$
\phi_{r}=f_{r} \phi_{i}
$$

and $\phi_{i}^{\prime}$ is the diffusely reflected fraction

$$
\phi_{i}^{\prime}=\left(1-f_{r}\right) \phi_{i}
$$

With this model, the power balance equation, equation (3) of part III.A., takes on the following form.

$$
\begin{aligned}
\rho_{v}^{\prime}\left(1+f_{r} \rho_{v}^{\prime}\right)(1 & -f) \rho_{t} \phi_{0} \\
& =\phi_{i}^{\prime} f+\left[(1-f) \phi_{i}^{\prime}+\phi_{r}\right]\left(1-\rho_{v}^{\prime}\right)
\end{aligned}
$$

where $\rho_{t}$ is reflectance of the target exclusive of the augmented reflection in the retro direction, which is lost through the port of the measuring instrument in every case. The relative reflectance $Q_{s}$ of the sphere is

$$
Q_{s}=k\left[\phi_{i}^{\prime} f\left(1-f^{\prime}\right)+\phi_{0}\left(1+f_{r} \rho_{v}^{\prime}\right) \rho_{t} f\left(1-f^{\prime \prime}\right)\right]
$$

and the relative reflectance of the target is, as before

$$
Q_{t}=k \phi_{0} \rho_{t}\left(1-f^{\prime}\right) .
$$

Equations (A2), (A3), (A4), (A5), and (A6) can be solved to obtain an expression for $\rho_{v}^{\prime}$ in the following form:

$$
\rho_{v}^{\prime}=\frac{1-f\left(1-f_{r}\right) \frac{Q_{t}}{Q_{s}}}{1-f\left(1-f_{r}\right)} \cdot \frac{1}{1-\alpha}
$$

where

$\alpha=\left\{\frac{f\left(1+\rho_{v}^{\prime} f_{r}\right)\left[f^{\prime}-f^{\prime \prime}\right]}{\left(1-f^{\prime}\right)}+\frac{f f_{r}\left(1-f_{r} \rho_{v}^{\prime 2}\right)}{1-\rho_{v}^{\prime}\left[1-f\left(1-f_{r}\right)\right]}\right\} \frac{Q_{t}}{Q_{s}}$

Comparing (A7) with (6), it is seen that $f$ in the dominant first term is replaced by $f\left(1-f_{r}\right)$. This in essence states that the fraction the flux escaping from the port has been reduced relative to the flux lost to the walls by an amount proportional to the retroreflected fraction. The correction term $\alpha$ for the singular treatment of the first target reflection when the target is in the auxiliary sphere is also modified by the retroreflectance, making the form of this term so complicated that a closed solution in terms of the measurement parameters is not practical. We solve the equation iteratively, using as a first approximation for $\rho_{v}^{\prime}$ the value obtained from (A7) with $\alpha=$ 0 . Taking the retroreflectance into account has a very small effect on the calculated value of $\rho_{v}^{\prime}$. For example, if $f_{r}=$ 0.001 , the effect of not ignoring this in the case of a sample for which $\rho_{v}^{\prime} \simeq 0.98$ is to reduce the calculated $\rho_{v}^{\prime}$ by approximately $10^{-6}$. Note that $\rho_{v}^{\prime}$ must be the total reflectance, including retroreflectance, since $1-\rho_{v}^{\prime}$ represents loss of radiation by absorption.

\section{Models for Retroreflective Mechanisms}

Our measurements of retroreflective bidirectional reflectance factor, the data for which are shown in figure 15 , were limited to observation angles greater than $0.1^{\circ}$. Therefore we sought a model which could be used to interpolate our data to $0^{\circ}$ observation angle so that we could evaluate the integral in eq (39).

A model based on shadowing was developed by Hapke [27] to explain the retroreflective phenomena observed from the lunar surface. This model, which was quite successful in Hapke's application, was used by Egan and Hilgeman [13] in an effort to quantitatively evaluate the retroreflectance which they observed from barium sulfate paint and other highly reflective white coatings. Other possible mechanisms for producing retroreflectance include cube corner reflectors formed from broken cubic crystals and retroreflectance by focussing, as occurs in glass beaded retroreflecting sheeting. In the following paragraphs we will treat special cases of each mechanism.

\section{a. Shadowing}

The Hapke model includes as one of its basic assumptions that the absorbance of the scattering particles is very high. In this way, there will be a considerable contrast between the radiance coming from shadowed areas in which the radiation undergoes several reflections and the radiance reflected from an unshadowed area. Although there is relatively little loss in highly reflecting materials such as barium sulfate or Halon, it is still possible for an initial first surface reflection to add to the background of multiply reflected radiation in a preferential manner. In order to estimate the order of magnitude of the non-diffuse reflection, one can use layers of close-packed spheres as a model for the many randomly oriented particles in the coating. Referring to one such sphere as shown in figure 18a, one can calculate the reflectance factor for such an array as follows. The bidirectional reflectance factor is given by

$$
F(\mathbf{U}, \mathbf{u})=\frac{\pi L(\mathbf{u})}{E(\mathbf{U})}
$$

where $E(\mathbf{U})$ is collimated irradiance incident in the direction $\mathbf{U}$ and $L(\mathbf{u})$ is the reflected radiance in the direction $\mathbf{u}$, both averaged over a sufficiently large area of the sample. In the case of retroreflectance, we set the unit vectors $\mathbf{U}$ and $\mathbf{u}$ equal. The average reflected radiance is given by definition as

$$
L(\mathbf{u})=\frac{\Phi(\omega, A)}{\omega(\mathbf{u}) A \cos \gamma} .
$$

where $\omega(\mathbf{u})$ is an element of solid angle oriented in the direction (u), $A$ is the area of the surface over which the average is being taken, and $\Phi(\omega, A)$ is the flux reflected from area $S$ into solid angle $\omega$. Collimated incident radiation will come to a virtual focus at a point $I$ which is $r / 2$ from the outer surface of the sphere. The solid angle $\omega$ is defined in terms of a small arbitrary area $a$ on the sphere

$$
\omega=\frac{4 a}{r^{2}} .
$$

The flux reflected from this area into $\omega$ is given by

$$
\Phi=\rho E a / \cos \gamma
$$


where $\rho$ is the reflectance of the sphere surface. (Note that since we are treating retroreflectance, $a$ is chosen so that it is centered on a radius in the direction of $E$, and therefore the direction of incidence is normal to $a$.) The area $A$ occupied by this sphere in a hexagonal close-packed array is

$$
A=\frac{6 r^{2}}{\sqrt{3}} .
$$

Using expressions (A9), (A10), (A11), and (A12) above in (A8) one obtains under conditions of retroreflectance:

$$
F(\overline{\mathbf{U}}, \mathbf{u})=\frac{\pi \sqrt{3}}{24} \rho / \cos ^{2} \gamma=0.227 \rho / \cos ^{2} \gamma .
$$

This would be roughly the maximum amount of additional bidirectional reflectance factor which might occur in the retro-direction under ideal conditions of shading by surrounding particles. The $\cos ^{2} \gamma$ term in the denominator is an artifact of the "array of spheres" model we are using. In the continuous surface being represented by the array of spheres model, the projected surface area goes as $\cos ^{2} \gamma$ so that the $\cos ^{2} \gamma$ term should be omitted.

\section{b. Cube corners}

In the ideal case, the bidirectional reflectance factor for a cube corner reflector would be infinite at zero observation angle and zero at all other observation angles. However, imperfections in the cube corners and diffraction tend to spread the reflected radiation out so that bidirectional reflectance factor has meaning in describing reflection by cube corners. For the model for maximum reflectance, we will assume that the cubes are perfect and that diffraction is the limiting factor. For near normal incidence the total retroreflectance from a close-packed array of cube corner reflectors is

$$
\rho_{t}=\rho_{1}^{3}\left(1-\rho_{2}\right)^{2}
$$

where $\rho_{1}$ is the reflectance of the cube faces and $\rho_{2}$ is the reflectance of the entrance surface. For a perfect internal corner $\rho_{1}$ is 1 and $\rho_{2}$ is roughly 0.05 , so that $\rho_{t}$ is roughly 0.9 . For an external corner (cube corner "hole"), $\rho_{2}=0$ and $\rho_{1}$ is roughly 0.05 so that $\rho_{t}$ is roughly $1.25 \times 10^{-4}$ in that case. The reflected radiance is distributed in the diffraction pattern associated with the reflecting faces treated as apertures. For this order of magnitude calculation, we will assume the pattern to be that of a circular aperture of effective radius $r=\sqrt{\frac{A}{\pi}}$ where $A$ is the area of the entrance surface. The radiance in such a pattern would be given by [28].

$$
L(\beta)=\frac{E A}{\lambda^{2}}\left[\frac{2 J_{1}(x)}{x}\right]^{2}
$$

where

$$
x=2 \pi r \sin (\beta) / \lambda,
$$

from which it follows that the reflectance factor is given by

$$
F(\beta)=\frac{\pi A}{\lambda^{2}}\left[\frac{2 J_{1}(x)}{x}\right]^{2} \rho_{t}
$$

The data in figure 15 suggest that the particle sizes are such that the central bright spot would subtend an angle of about $1^{\circ}$ for $\mathrm{BaSO}_{4}$. The edge of the central bright spot falls at $x=$ 3.8. For an effective wavelength of approximately $550 \mathrm{~nm}$, it follows that the particle size would have to be such that $r \simeq$ $20 \mu \mathrm{m}$. Thus, the reflectance factor at $\beta \ll 1^{\circ}$ would be roughly

$$
F(\beta) \simeq 10^{4} \rho_{t}
$$

\section{c. Focussing retroreflectors}

If the index of refraction of a bead is such that the radiation passing through it comes to a focus behind it, a suitable reflector may be placed at the focal point (I in fig. 18c.) and the radiation will be reflected back through the bead into a collimated retroreflected beam. Here, the spreading of the reflected radiance can be brought about either by diffraction, poor focussing, or both. The diffraction limiting case in the previous paragraph can be applied to the Halon data in figure 15. In this case, the central bright spot appears to subtend an angle of about $0.5^{\circ}$ which corresponds to an effective lens radius of $r \simeq 40 \mu \mathrm{m}$. If one assumes a refractive index of 1.5 so that the radiation will come to a focus on the back of the bead and from experience with cheap cameras assumes that

a.

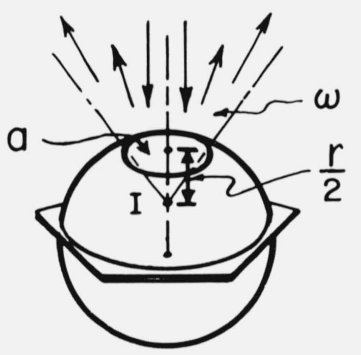

b.

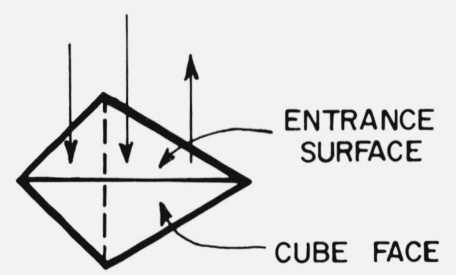

c.

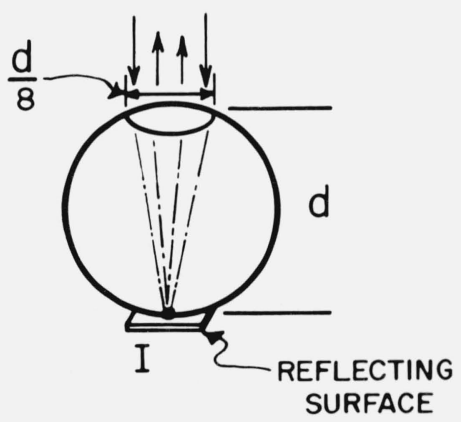

FIGURE 18. Diagrams for estimating magnitude of retroreflectance by various means. 
the diameter of the effective lens area is roughly one-eighth of the diameter of the bead, it follows from expressions (A12) and (A16) that a closely packed array of such spheres would have a reflectance factor for $\beta \ll 1$ of

$$
F(\beta) \simeq 6 \times 10^{2} \rho_{t}
$$

for a specular reflector of reflectance $\rho_{t}$ located at I and

$$
F(\beta) \simeq 10 \rho_{t}
$$

for a diffuse reflector of reflectance $\rho_{t}$ at $\mathrm{I}$.

\section{Interpolating the retroreflectance data}

From the foregoing analysis, one can make the following deductions, assuming that the 0.5 excess reflectance factor is caused by only one mechanism:

a. Shadowing cannot by itself account for the observed retroreflectance.

b. At least $6 \times 10^{-5}$ of the area is effectively taken up with inside corner cube retroreflectors.

c. At least 0.4 of the area is effectively taken up with outside corner cube retroreflectors.

d. At least $1.6 \times 10^{-2}$ of the area is effectively taken up with focusing sphere retroreflectors backed by specular reflectors of reflectance 0.05 .

e. At least $5 \times 10^{-2}$ of the area is effectively taken up with sphere retroreflectors backed by diffuse reflectors of reflectance 1 .

Since the mechanisms are not mutually exclusive, it is expected that all will operate to some extent in pressed powder samples. However it is highly unlikely that cube corner reflectors will be formed in Halon, so it would be expected that the mechanism in $\mathrm{d}$. or e. above would predominate. Even though the cleavage of a crystal in such a way that it forms an inside corner cube reflector is not very likely, mechanism b. probably predominates in the $\mathrm{BaSO}_{4}$ powder. Shadowing will contribute some effective retroreflectance in both cases.

The predominate mechanisms appear to depend on the sum of the diffraction patterns associated with a number of reflectors, each with a different effective aperture, and the statistical distribution of the aperture sizes is unknown. Therefore we chose to use an emperical fitting function. The function

$$
F(\beta)=1+\exp \left(a+b \beta^{c}\right)
$$

was found to fit the data adequately. Fitting all $\mathrm{BaSO}_{4}$ data, we obtained

$$
\begin{aligned}
& a=-0.393 \\
& b=-0.966 \\
& c=0.57
\end{aligned}
$$

and fitting the first seven points of the Halon data we obtained

$$
\begin{aligned}
& a=1.18 \\
& b=-3.41 \\
& c=0.21
\end{aligned}
$$

These fitting functions are shown as solid curves in figure 15 . The average of the function $F(\beta)$ for $\mathrm{BaSO}_{4}$ over the range $0 \leq \beta \leq 0.5^{\circ}$ is 1.42 which is in good agreement with the data of Egan and Hilgeman [13]. The difference values for $\rho\left(6^{\circ}, \lambda\right)-F\left(6^{\circ}, \lambda\right)$ were obtained by using the fitted functions in eqs (39) and (40) with $r=1.9 \mathrm{~cm}$ and $d^{\prime}=29.4 \mathrm{~cm}$. In each case, the contribution from the region of extrapolation $\left(|\beta|<0.1^{\circ}\right)$ was only a small part of the total flux in the retroreflected component, so the choice of fitting functions was not critical. A crude measurement of $\mathrm{BaSO}_{4}$ using a beam splitter yielded $\rho\left(6^{\circ}, \lambda\right)-F\left(6^{\circ}, \lambda\right)=0.0006$ which is in good agreement with the value of 0.0007 obtained from the data in figure 15.

\section{Appendix B. Interpretation of Error Statements}

The error analysis in this paper involves three distinct classes of errors. The first class of error is the measured random error. This type of error appears as noise in the measurements and is evaluated by making each measurement several times and performing the usual error analysis on the results. For this type of error, we will cite three times the standard deviation of the mean.

The second class of error is the estimated random error. This class of error contributes to the outcome in the same way as the measured random error, but, either because of the inconsequential effects of the error or because of experimental difficulties which would be involved in evaluating it, we choose to estimate the size of the error instead of determining it by making several measurements. In making such an estimate, we choose to estimate the smallest range of uncertainty within which the correct value will fall with a very high probability, i.e., an intuitive ninety-five times out of a hundred. For this type of error, we will cite the size of the estimated error itself. The estimation process is carried out in such a way that the sign of the error is indeterminant. If it is known that a particular cause of error will result in an error which is always of one sign, we will correct the measured value to a most probable value and reduce the magnitude of the estimated random error appropriately. The estimated random error is combined in quadrature with the measured random error to obtain the total random error. All random errors, whether measured or estimated, will be denoted by the symbol $\delta$.

The third type of error is the estimated systematic error, indicated by the symbol $\Delta$. This error is like the estimated random error in all respectes except one, namely it is not independent from determination to determination, i.e., it will affect all determinations in the same way. It should be pointed out that this does not mean that the sign of the measurement is known, but only that the sign of the effect is known to be fixed from determination to determination. For example, in correcting for the reflection of radiation from the beveled lip of the sphere, we estimate that the radiation will be reflected with an average reflectance intermediate between one and the normal reflectance of the stainless steel. (See discussion preceding (19).) If the actual reflectance is Tess than our estimated value, determinations of reflectance made using this estimate will result in $\rho_{v}$ values which are all slightly higher than if the estimates were correctly made. In the course of many determinations, the total random error will be reduced, but the systematic error will remain. Since the 
sign of the systematic errors is not known, systematic errors from independent causes will be added in quadrature, and at each point in the analysis at which it is desirable to estimate a total uncertainty, the systematic error and the total random error will be added in quadrature. However, when proceeding to combine the results of several determinations, we will first combine the random errors in the ordinary way and add the largest systematic error in quadrature at the end. In this way, the calculated systematic error is not reduced by repeating the measurements. The total uncertainties so calculated are to be regarded as determining a range within which we expect the correct value to fall with a probability of approximately 0.95 . This total uncertainty will be indicated by the symbol $\epsilon$.

\section{Appendix C. Calculating $C(\Gamma, \lambda)$}

The integrals in (11) are evaluated in closed form after fitting the experimental data for $N_{g}$ and $N_{G}$.

\section{Fitting $N_{G}(\Gamma, \lambda)$}

The data for $N_{G}$ were fitted by an expression of the form:

$$
N_{G}(\Gamma, \lambda)=a_{0}(\lambda)+a_{1}(\lambda) \Gamma^{2}+a_{2}(\lambda) \Gamma^{4} .
$$

An even function of $\Gamma$ was used, since we have assumed the directional-hemispherical reflectance of the coatings does not depend on the angle of azimuth of the incident direction, but depends only on the angle of elevation. The coefficients in (C1), as determined by a least squares fitting of the experimental data are given in table C1.

\section{Fitting $N_{g}(\Gamma, \lambda)$}

Since the experimental data for $N_{g}(\gamma, \lambda) / \cos (\gamma)$ were found to be nearly independent of wavelength, only one function $N_{g}(\Gamma)$ is needed for each type of coating. However, since there was such a large uncertainty in the $N_{g}(\Gamma)$ data, three sets of $N_{g}(\Gamma)$ were fitted as described in part IV.B.3. in the main text. The form used for the fitting the data was:

$$
N_{g}(\Gamma) / \cos \Gamma=\sum_{i=0}^{4} b_{i} \Gamma^{i}
$$

TABLE CI

Coefficients for the expansion of $N_{G}(\Gamma, \lambda)$ in even powers of the angle of incidence $\Gamma$ in radians at three different wavelengths for three sphere wall coatings.

\begin{tabular}{ccccc}
\hline \hline Coating & $\begin{array}{c}\text { Wave- } \\
\text { length }\end{array}$ & \multicolumn{1}{c}{$a_{0}$} & $\begin{array}{c}a_{1}{ }^{*} \\
\times 10^{3}\end{array}$ & $\begin{array}{c}a_{2}{ }^{*} \\
\times 10^{3}\end{array}$ \\
\hline \multirow{3}{*}{ BaSO $_{4}[14]$} & 450 & 0.999942 & 5.99 & 0.438 \\
& 550 & .99978 & 4.40 & 0.159 \\
& 750 & 1.00011 & 3.27 & -0.00814 \\
Halon [15] & 450 & 0.999875 & 3.97 & -0.172 \\
& 550 & 1.00004 & 4.17 & -0.985 \\
& 750 & 1.00027 & 3.68 & -1.20 \\
BaSO $_{4}[16]$ & 450 & 0.999829 & 4.08 & 0.405 \\
& 550 & 1.00047 & 1.43 & 0.741 \\
& 750 & 1.00012 & 3.91 & -0.471 \\
\hline
\end{tabular}

* The second and third digits are not significant but are kept to avoid round-off error in further calculations.
In the case of the original knife-edge data, the values of the coefficients, as obtained by least squares fitting, are given in table CII as describing the "upper limit" curve. In the original data, the least uncertainty was associated with the $\Gamma=0$ reading. Therefore, all data were normalized to the $\Gamma$ $=0$ reading and $b_{0}$ was taken to be 1 when the data were fitted. Because of the symmetry in azimuth, $b_{1}$ and $b_{3}$ are taken to be zero.

In the accepted data, $N_{g}(\Gamma) / \cos \Gamma$ was taken as 1 up to $\Gamma=$ $\pi / 4$. To obtain the remaining part of this curve we obtained $N_{g}(\gamma)$ according to the expression

$$
N_{g}^{\prime}(\gamma) / \cos \gamma=1+C_{1} \gamma
$$

The difference data $N_{g}(\gamma)-N_{g}^{\prime}(\gamma)$ using the data for the angles $\pi / 3\left(60^{\circ}\right), 5 \pi / 12\left(75^{\circ}\right)$ and $17 \pi / 36\left(85^{\circ}\right)$ were fitted using the form

$$
N_{g}(\gamma) /(\cos \gamma)=1+b_{1}^{\prime}\left(\Gamma-\frac{\pi}{4}\right)^{2}+b_{2}^{\prime}\left(\Gamma-\frac{\pi}{4}\right)^{4}
$$

After $b_{1}^{\prime}$ and $b_{2}^{\prime}$ were determined, these were used to obtain the $b_{i}$ in the expression (C2).

\section{Calculating $C(\boldsymbol{\Gamma}, \lambda)$}

With the expressions (C1) for $N_{G}(\Gamma, \lambda)$ and (C2) for $N_{g}(\Gamma$, $\lambda)$ substituted into eq (11), the integrals in that equation can be evaluated in closed form in terms of integrals of the form

$$
I_{i}=\int_{0}^{\pi / 2} \Gamma^{i} \cos \Gamma \sin \Gamma d \Gamma
$$

for the upper and lower limit functions, and

$$
I_{i}^{\prime}=\int_{0}^{\pi / 4} \Gamma^{i} \cos \Gamma \sin \Gamma d \Gamma
$$

and

$$
\mathrm{I}_{i}^{\prime \prime}=\int_{\pi_{14}}^{\pi / 2} \Gamma^{i} \cos \Gamma \sin \Gamma d \Gamma
$$

for the accepted value functions. The values of these integrals are given in table CIII, and the resulting values of $\bar{N}_{G}(\lambda)$ are given in table CIV. It can be seen from table CIV that the difference between the various assumptions concerning the self-radiance has a very small effect on the value of $\bar{N}_{G}$ in all cases. Therefore only the accepted value of $\bar{N}_{G}$ will be used in each case to calculate $C(\Gamma, \lambda)$. The results of the scanning which has been done to date with the telescopic detector indicate that the real value probably lies toward the lower limit from the accepted value. On this belief, we will assign the largest difference between an accepted value and a lower limit value, 0.00005 , as the magnitude of the uncertainty in $\bar{N}_{G}$ due to the uncertainty in evaluating the self-radiance by measuring $N_{g}$.

\section{Appendix D. The Three Working Standards}

The properties which standards for reflectance factor measurements should have is a subject over which there is much diversity of opinion. By definition, reflectance factor is reflectance relative to the reflectance of a totally reflecting Lambertian reflector, both measured on the same instrument. Based on this definition, the best standard for calibrating an 
TABLE CII

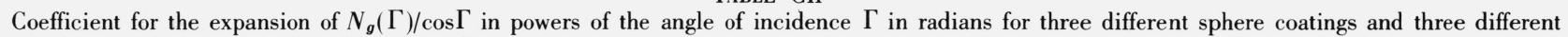
assumptions concerning the self-radiance distribution.

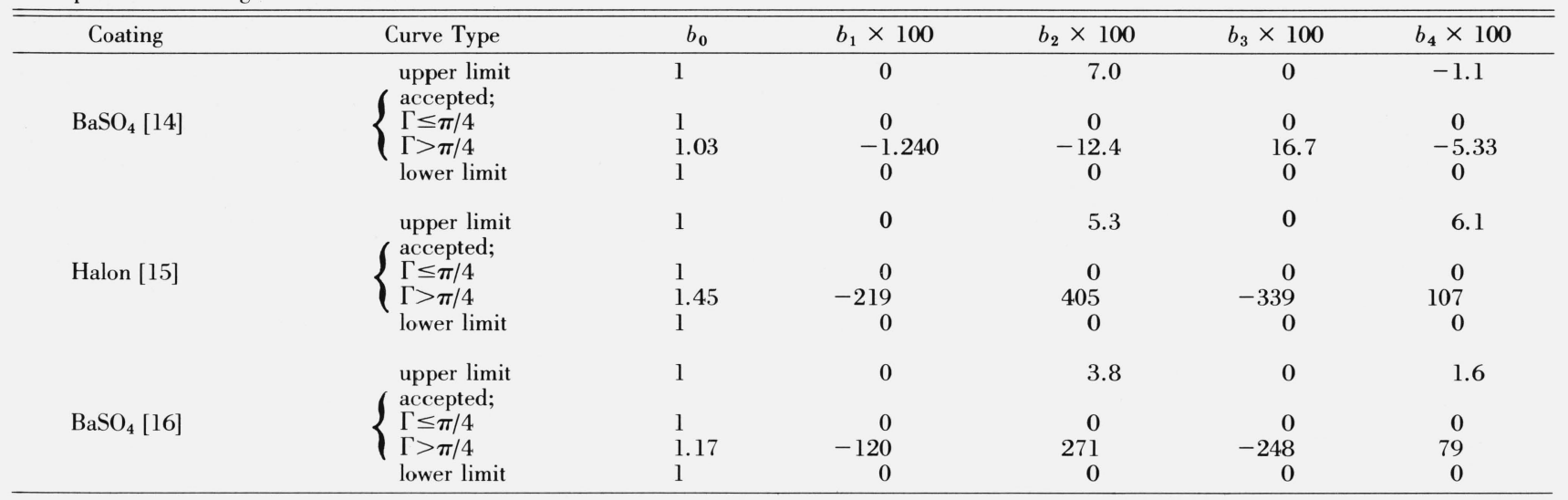

TABLE CIII

Values of the integrals in equations (C5), (C6), and (C7) for nine values of

\begin{tabular}{cccc}
\hline \hline$i$ & $I_{i}$ & $I_{i}{ }^{\prime}$ & $I_{i}{ }^{\prime \prime}$ \\
\hline 0 & 0.50000 & 0.25000 & 0.25000 \\
1 & .39270 & .12500 & .26770 \\
2 & .36685 & .07135 & .29550 \\
3 & .37990 & .04382 & .33608 \\
4 & .42147 & .02819 & .39328 \\
5 & .49129 & .01872 & .47257 \\
6 & .59443 & .01273 & .58170 \\
7 & .74046 & .00882 & .73164 \\
8 & .94416 & .00614 & .93802 \\
\hline
\end{tabular}

TABLE CIV

Values of the weighted averages $\bar{N}_{G}(\lambda)$ for each of three coatings for three wavelengths and three different assumptions concerning the self-radiance distribution.

\begin{tabular}{ccccc}
\hline \hline $\begin{array}{c}\text { Determination } \\
\text { number }\end{array}$ & $\begin{array}{c}\text { Wave- } \\
\text { length } \\
\lambda\end{array}$ & Upper limit & $\begin{array}{c}\text { Accepted } \\
\text { value }\end{array}$ & Lower limit \\
\hline BaSO $_{4}[14]$ & 450 & 1.00486 & 1.00479 & 1.00476 \\
& 550 & 1.00343 & 1.00338 & 1.00336 \\
& 750 & 1.00244 & 1.00240 & 1.00239 \\
Halon [15] & 450 & 1.00294 & 1.00282 & 1.00277 \\
& 550 & 1.00233 & 1.00225 & 1.00223 \\
& 750 & 1.00175 & 1.00170 & 1.00169 \\
& 450 & 1.00343 & 1.00335 & 1.00333 \\
$\mathrm{BaSO}_{4}[16]$ & 550 & 1.00173 & 1.00168 & 1.00167 \\
& 750 & 1.00253 & 1.00247 & 1.00247 \\
\hline
\end{tabular}

instrument to measure reflectance factor would be a totally reflecting Lambertian reflector, or the closest thing to it which could be obtained. The Halon and barium sulfate sphere linings which were used in the work described in this paper were very close to this ideal.
However, if reflectance factor measurements are going to be of use in practical applications, the instrument must be defined as part of the specification of the measurement. It is for this reason that we have defined the measurements as carefully as possible in terms of integrals describing the incident radiation and the instrument response. We believe the properties of our reference reflectometer are very close to those specified in the definition for directional-hemispherical reflectance factor which we have used, and furthermore, we believe the geometrical properties of that instrument to be stable in time. In order to confirm this, however, we feel that it is important to have several working standards representing the gamut of the highly reflecting materials which we would be measuring with this instrument. It is also important that several different types of working standards be used in order to be able to detect changes in the optical properties of any one of them. As a means of retaining our scale of measurement in day-to-day measurements and as a means of comparing one determination with another, we are using the following working Standards:

Working Standard No. 1-This standard is a piece of MC20 opal glass purchased in September 1970 from

$$
\begin{aligned}
& \text { Mashpriborintorg } \\
& \text { Smolenskaja pl., 32/34 } \\
& \text { 121200, Moscow, G-200 } \\
& \text { U.S.S.R. }
\end{aligned}
$$

The piece used is $99 \mathrm{~mm}$ by $99 \mathrm{~mm}$ and is $20 \mathrm{~mm}$ thick. It is marked MC-20-2 for identification and is usually referred to as the Russian Opal Glass. The side which is measured has a very flat, highly polished surface.

Working Standard No. 2-This standard is a piece of Vitrolite glass which was manufactured by

Libbey Owens Ford
1701 E. Broadway
Toledo, Ohio 43605

The exact date of manufacture of this particular piece of glass is unknown, but it has been at least twenty years since any glass of this type has been manufactured. The piece used is 
$100 \mathrm{~mm}$ by $100 \mathrm{~mm}$ and is $11 \mathrm{~mm}$ thick. It is marked V6-D1 for identification. The side which is measured is flat and highly polished.

Working Standard No. 3-This standard is a porcelain enamel on steel plaque which was made around 1946 by

The Harshaw Chemical Co.

Division of Kewanee Oil Company

1933 E. 97th St.

Cleveland, Ohio 44106

The plaque used is $108 \mathrm{~mm}$ by $108 \mathrm{~mm}$ and is marked 80-1 for identification. The surface which is measured is smooth and non-porous, but it has a slight ripple or "orange peel" texture.

These three working standards cover the range of reflectance factor from nearly 1 to approximately 0.8 , which is the range generally used for standards to calibrate the gain setting of reflectometers. In addition, the Vitrolite standard is slightly translucent. Recent tests have shown our instrument to be insensitive to this amount of translucence, but if this should change the difference will be noticeable in the mea- surements obtained using this standard as compared to the others. Because of the rippled surface in the porcelain enamel standard, the surface reflection from this standard is spread out more than that from the other two standards which have flat surfaces. Therefore, differences in the way the instrument handles specular reflection may show up in the relative measurements of this standard with respect to the others. (The most sensitive test of the way the instrument treats specular reflectance is made by measuring a specular mirror. We intend to use this procedure as soon as we have the capability to measure the reflectance of a mirror with the necessary accuracy.)

The cleaning procedure used is an important part of maintaining working standards of reflectance. Our present approach is to use a procedure which will disturb as little as possible the layers of oxides and other surface films characteristic of the materials. Therefore, we store the standards in dessicators and before each measurement we wash them with a mild nonfluorescing soap, rinse them thoroughly with hot water, rinse them with distilled water, and blot them dry with soft tissue paper. Any lint which remains on the surface is removed by gently brushing with a soft brush. 